This item was submitted to Loughborough's Research Repository by the author.

Items in Figshare are protected by copyright, with all rights reserved, unless otherwise indicated.

\title{
An investigation of a high-speed ground manoeuvre under optimal control
}

\section{PLEASE CITE THE PUBLISHED VERSION}

https://doi.org/10.1177/0954410018821793

PUBLISHER

SAGE Publications @ IMechE

\section{VERSION}

AM (Accepted Manuscript)

\section{PUBLISHER STATEMENT}

This work is made available according to the conditions of the Creative Commons Attribution-NonCommercialNoDerivatives 4.0 International (CC BY-NC-ND 4.0) licence. Full details of this licence are available at: https://creativecommons.org/licenses/by-nc-nd/4.0/

\section{LICENCE}

CC BY-NC-ND 4.0

\section{REPOSITORY RECORD}

Huang, Zexin, Matt Best, and James Knowles. 2018. "An Investigation of a High-speed Ground Manoeuvre Under Optimal Control”. figshare. https://hdl.handle.net/2134/36401. 


\section{An Investigation of a High-speed Ground Manoeuvre Under}

\section{Optimal Control}

This paper studies the behaviour of a nonlinear aircraft model under optimal control for aircraft ground manoeuvres, specifically for high-speed runway exits. The aircraft's behavior on the ground is captured by a fully parameterized 6-DOF nonlinear model. A predefined cost function is minimized using a Generalized Optimal Control (GOC) algorithm, in order to obtain an optimal control sequence for a particular manoeuvre-cost function combination. In this paper, three scenarios are investigated for a 45-degree high-speed runway exit: the first control sequence minimizes the distance between the aircraft's CG and the runway centreline; the second maximizes the distance travelled by the aircraft during the 20 seconds of simulation time; the third minimizes tire wear. For each scenario, the GOC algorithm provides the best possible control inputs: such results provide a benchmark against which the effectiveness of future real-time controllers may be judged.

\section{Nomenclature}

$V_{x}, V_{y}, V_{z} \quad=$ aircraft translational velocities along each of the aircraft body axes

$W_{x}, W_{y}, W_{z} \quad=$ aircraft angular velocities about each of the aircraft body axes

$F_{x N}, F_{y N}, F_{z N} \quad=$ force elements on the nose gear in the local tire coordinate system

$V_{x N}, V_{y N}, V_{z N}=$ nose gear velocities in the local tire coordinate system

$F_{x R}, F_{y R}, F_{z R} \quad=$ force elements on the right gear in the local tire coordinate system

$V_{x R}, V_{y R}, V_{z R} \quad=$ right gear velocities in the local tire coordinate system

$F_{x L}, F_{y L}, F_{z L} \quad=$ force elements on the left gear in the local tire coordinate system

$V_{x L}, V_{y L}, V_{z L} \quad=$ left gear velocities in the local tire coordinate system

$F_{x T L}, F_{x T R} \quad=$ right and left thrust force parallel to aircraft's X-axis

$F_{x A}, F_{y A}, F_{z A} \quad=$ aerodynamic force elements at the aerodynamic center of the aircraft 


$$
\begin{array}{ll}
M_{x A}, M_{y A}, M_{z A} & =\text { aerodynamic moment elements at the aerodynamic center of the aircraft } \\
F_{z W} & =\text { weight of the aircraft at the center of gravity } \\
X, Y, Z & =\text { global position of aircraft center of gravity } \\
\psi, \theta, \varphi & =\text { yaw angle, pitch angle and roll angle of the aircraft } \\
V_{x G}, V_{y G}, V_{z G} & =\text { aircraft angular velocities in the ground coordinate system } \\
W_{x G}, W_{y G}, W_{z G} & =\text { deflection of each tire in the ground coordinate system } \\
\delta_{z N}, \delta_{z R}, \delta_{z L} & =\text { slip ratio of the right and left gear } \\
S_{R, L} & =\text { normalized slip ratio of the right and left gear } \\
\bar{S}_{R, L} & =\text { normalized slip angle of the right and left gear } \\
\alpha_{N, R, L} & =\text { longitudinal stiffness of the right and left gear } \\
\bar{\alpha}_{R, L} & =\text { cornering stiffness of the right and left gear } \\
C_{x R, L} & =\text { longitudinal friction coefficient of the right and left gear } \\
C_{y R, L} & \text { wheel angular velocity of the right and left gear } \\
\mu_{x R, L} & =1 \text { right and left gear } \\
\mu_{y R, L} & =1 \text { friction coefficient of the right and left gear } \\
\Omega_{R, L} &
\end{array}
$$

\section{Introduction}

Despite recent advances in autonomous systems capabilities, aircraft ground manoeuvres are still conducted manually, leaving this phase of an aircraft's journey vulnerable to human error. A recent study of commercial aviation accidents identified that $11 \%$ of all fatal accidents occurred when the aircraft was on the ground [1]. Other non-fatal ground-based incidents typically lead to long delays, costing airlines money as planes are grounded for longer than planned. Despite the impact of fatal and non-fatal ground-based incidents, they still occur frequently: the International Air Transport Association (IATA) recorded 50 accidents over the period 2010-2014 [2]; the International Civil Aviation Organization (ICAO) found that $90 \%$ of scheduled commercial air transport accidents from 2008-2016 were runway-based. Reliable automation of runway manoeuvres could therefore help to reduce this significant contributor to aviation incidents. 
Whilst autopilot systems have been commonly used by aircraft in flight, the control of aircraft when on the ground has received limited attention in the literature. A yaw rate control law based on dynamic inversion and feedback linearization is presented in [5], where aircraft yaw rate was constrained by a linear controller to follow a pre-defined path. To cope with nonlinearities inherent in tyre/ground friction forces, tyre behaviour was approximated with a saturation nonlinearity. An anti-windup control strategy is also proposed in the literature as a method for controlling an aircraft's steer angle when on the ground [6][7]. The model used in this work was a linear parameter variable (LPV) aircraft model (based on a bicycle model) [7], where tyre/ground friction forces were also approximated with a saturation nonlinearity. Simulation results showed that the control method works for low-speed lateral manoeuvres, a region where the tyre approximation is likely to be reasonably valid. Whilst the saturation nonlinearity approximation is better than a purely linear tyre model (because there is a point where the tyre cannot generate any further lateral force) the nature of the saturation does not capture real tyre behaviour correctly, so the dynamics at slip angles near the saturation point will not be correct. This means that the controllers proposed in the literature may struggle to cope with high-speed manoeuvres, where tyre slip angles may operate in the region around the saturation point.

Part of the challenge facing researchers working to control aircraft ground manoeuvring is that there is a limited number of models that have been developed in the literature. Rankin et. al. present a nonlinear 6-DOF model of an aircraft's ground dynamics [4], where nonlinearities are included in the lateral tyre dynamics, aerodynamics and shock absorber dynamics. The model is derived from the GATEUR model [18], an industrially-validated Simulink model that is known to produce accurate representations of aircraft ground dynamics. Rankin et. al. use their model to conduct a comprehensive bifurcation analysis of the aircraft's lateral dynamics [3], highlighting how boundaries associated with the lateral stability of steady-state turning solutions vary with operational parameters such as thrust level and c/g position. The bifurcation results provide a comprehensive picture of the underlying steady-state dynamics, but provide little information about the transient behaviours that may be experienced when entering or exiting from a turn manoeuvre. In a development of their work, Rankin et. al. present a general approach to evaluate lateral loads experienced by individual landing gears in transient conditions, for two different manoeuvres - a $45^{\circ}$ high speed runway exit, and a $90^{\circ}$ runway exit - and two aircraft weights [4]. The method parameterized the runway turn in terms of an approach velocity, and used a hyperbolic tangent function for the steering input (to approximate 
how a pilot might approach the turn). It was assumed that all braking occurred to achieve the desired initial velocity before initiating the turn, so the results only considered lateral dynamic effects.

The aircraft model used in this work to obtain optimal control strategies is based on Rankin's 6-DOF model. The applicability of control strategies to real-life applications depends heavily on the quality of model used. The simplified models used in previous works to demonstrate control techniques [5-7] have limitations when considering high lateral accelerations, whereas Rankin's model is designed to capture these nonlinearities accurately. In order to apply both braking and steering inputs, this work presents a development of Rankin's model, through the inclusion of a combined-slip tire model: when cornering, the tyre develops a lateral slip angle (between the plane of wheel rotation and the direction of wheel travel) due to turning; when braking, the tyre develops a longitudinal slip ratio (between the wheel's contact-patch speed and its translational speed); the normalized total slip is introduced to calculate the total tyre force generated by combined braking and steering. This total tyre force behaves like the individual lateral and longitudinal slips - it builds up rapidly (from zero force at zero slip) before reaching maximum total force at the optimal slip value. As the total slip continues to increase, the tire saturates which results in a reduction of the total tire force generated. The application of longitudinal slip reduces the lateral force that can be generated at a given slip angle condition, and conversely the application of (lateral) slip angle reduces the longitudinal force at a given braking condition - hence why racing drivers typically brake before a corner. Because the braking and steering system on most aircraft are typically independent (steering actuator on nose-wheel whilst braking actuator on main-gears), such a strategy may not produce an optimal control sequence for runway exit manoeuvres. Instead, the Generalized Optimal Control (GOC) algorithm used in this paper will determine the maximum required brake torque and steer inputs that can be applied, whilst still negotiating the runway exit.

Unlike the work by Rankin et. al., there are no pre-defined steer inputs used in this current work: the optimal control inputs are defined with regard to a specified cost function, with the aircraft's dynamics providing realistic limitations to steer and braking. Here, an iterative simulation-based indirect method of numerical optimization known as Generalized Optimal Control (GOC) [8] [9] is used to identify the optimal control inputs, i.e. control inputs that minimize a user-defined cost function. These time-variant control inputs (e.g. steering, braking, thrust) can be optimized simultaneously for any smoothly nonlinear system, as demonstrated in a recent study of collisionavoidance strategies for cars [10]. Some direct methods of numerical optimization such as nonlinear programming (NLP) have been used to optimize aircraft trajectories [18][24][25]. Rather than integrating the cost along the 
trajectory from initial condition, a direct method employs collocation method or pseudo spectral method to approximate the integral of cost function. By doing this, the optimal control problem is transcribed to a Non-Linear Programming problem which can be then solved with a NLP solver. In direct methods, constraints on states and control are imposed explicitly, while in GOC the constraints are introduced as continuous cost components represented by nonlinear functions, e.g. 'tanh' function. Hard constraints are not considered in this paper because GOC is used to control nonlinear smooth systems, which means the state-space equations must be differentiable over the fixed time interval $[0 \mathrm{~T}]$. Although direct methods are popular and widespread especially in practice, GOC is still proposed in this study because the emphasis of this paper is an off-line numerical investigation of optimal ground manoeuvres. Additionally, GOC shows good convergence to optimal solution in all the control problems considered in this paper.

This paper considers an aircraft performing a high-speed ground manoeuvre - a 45-degree runway exit. For a specified initial condition without loss of generality, the optimal control sequence varies with different objectives expressed in the composition of the cost functions. Three scenarios are considered here; the first scenario determines the control sequence that minimizes the distance between the aircraft's c/g and the runway centreline; the second scenario determines the control sequence that maximizes the distance travelled by the aircraft during the 20 seconds of simulation time; the final scenario determines the control sequence that minimizes tire wear. The paper is presented as follows: Section 2 introduces the mathematical model used in these studies, including the creation of a combined slip tyre model; Section 3 outlines the GOC algorithm; Section 4 presents the optimal control sequences for the three cases considered; Section 5 provides some concluding remarks, highlighting considerations for practical implementation of the runway exit manoeuvre and some directions for future work.

\section{Aircraft Model}

This section introduces the mathematical aircraft ground dynamics model used in this work. The model is an implementation of a nonlinear model presented in [4], which was developed from an industrially-validated SIMMECHANICS model [21]. It is therefore known to provide physically-relevant behaviour of an aircraft's ground dynamics. In the model, the airframe is assumed to act as a rigid body with six degrees-of-freedom (DOF), with a coordinate system consistent with the aircraft's principle axes of inertia: the origin is the center of gravity; the $\mathrm{x}$-axis is positive towards the nose of the aircraft; the $\mathrm{z}$-axis is positive towards the ground; the $\mathrm{y}$-axis is positive 
starboard (as defined by the right hand rule). The airframe interfaces with the ground via three landing gears. Two main gears are connected to the airframe with translational joints (allowing a relative translation along the vertical axis only), whilst the nose gear is connected with a cylindrical joint (allowing a relative translation along and a rotation about the vertical axis). The model includes nonlinear effects in: the aerodynamics; the tire/ground forces; the main landing gear shock absorber compression/extension dynamics. Whilst the model's parameters can be chosen to represent any passenger aircraft, the values used in this study (obtained from the literature in [4]) represent a mid-sized passenger aircraft.

Since this paper aims to optimize the application of steering, braking and thrust simultaneously, the model from [4] has been developed with addition of a combined-slip tire model. The brakes, which are only applied to the maingears, create a difference between the wheel's contact-patch speed and translational speed. This requires another two state variables in the model, to represent the main-gears' contact-patch velocities. The control variables considered here include steering angle $\delta$, thrust force of the right/left engine $F_{x T R} / F_{x T L}$, and brake torque $T_{\text {brake }}$. The following subsections provide details of the mathematical model, highlighting developments that have been made in this paper.

\section{A. Equations of Motion}

The aircraft's equations of motion are formulated as a coupled system of ordinary differential equations (ODEs), formed by balancing forces and moments in each degree of freedom [12]. Additional state coupling occurs via relative sub-models, which provide expressions for quantities such as the aerodynamic forces in the aircraft's xdirection $\left(F_{x A}\right)$. The model states are the aircraft's translational and rotational velocities in the local body coordinate system, which are used to form six ODEs [4]:

$$
\begin{array}{rll}
m\left(\dot{V}_{x}+V_{y} W_{z}-V_{z} W_{y}\right)= & F_{x T L}+F_{x T R}-F_{x R}-F_{x L}-F_{x N} \cos (\delta)-F_{y N} \sin (\delta)-F_{x A}+F_{z W} \sin (\theta) \\
m\left(\dot{V}_{y}+V_{x} W_{z}-V_{z} W_{x}\right)= & F_{y R}+F_{y L}+F_{y N} \cos (\delta)-F_{x N} \sin (\delta)+F_{y A}+F_{z W} \sin (\varphi) \\
m\left(\dot{V}_{z}+V_{y} W_{x}-V_{x} W_{y}\right)= & F_{z W} \cos (\theta) \cos (\varphi)-F_{z R}-F_{z L}-F_{z N}-F_{z A} \\
I_{x x} \dot{W}_{x}-\left(I_{y y}-I_{z z}\right) W_{y} W_{z}= & l_{y L} F_{z L}-l_{y R} F_{z R}-l_{z L} F_{y L}-l_{z R} F_{y R}-l_{z N} F_{y N} \cos (\delta)+l_{z N} F_{x N} \sin (\delta) \\
& +l_{z A} F_{y A}+M_{x A} \\
I_{y y} \dot{W}_{y}-\left(I_{z z}-I_{x x}\right) W_{x} W_{z}= & l_{x N} F_{z N}-l_{z N} F_{x N} \cos (\delta)-l_{z N} F_{y N} \sin (\delta)-l_{x R} F_{z R}-l_{z R} F_{x R}-l_{x L} F_{z L}- \\
& & l_{z L} F_{x L}+l_{z T} F_{x T R}+l_{z T} F_{x T L}+l_{z A} F_{x A}+l_{x A} F_{z A}+M_{y A} \\
I_{z z} \dot{W}_{z}-\left(I_{x x}-I_{y y}\right) W_{x} W_{y}= & l_{y R} F_{x R}-l_{y L} F_{x L}-l_{x R} F_{y R}-l_{x L} F_{y L}+l_{x N} F_{y N} \cos (\delta)-l_{x N} F_{x N} \sin (\delta)+
\end{array}
$$




$$
l_{x A} F_{y A}+l_{y T} F_{x T L}-l_{y T} F_{x T R}+M_{z A}
$$

Two additional ODEs describe the two main-gears' wheel rotations using a moment balance between the brake torque $T_{\text {brake }}$ and friction force $F_{x R, L}$ about the wheel's roll axis:

$$
\begin{gathered}
I_{M G} \dot{W}_{R}=-F_{x R} * R_{\text {wheel }}-T_{\text {brake }} \\
I_{M G} \dot{W}_{L}=-F_{x L} * R_{\text {wheel }}-T_{\text {brake }}
\end{gathered}
$$

The model parameters chosen for this work are given in Table 1. The term ' $\mathrm{CG}$ ' is the location of the centre of gravity from the leading edge of the wing root, expressed as a percentage of Mean Aerodynamic Chord (MAC). In this paper, the center of gravity is fixed at its forward position of $14 \% \mathrm{MAC}$, which corresponds to a lightweight mass case $(45420 \mathrm{~kg})$. The aircraft's weight $\left(F_{z W}=m g\right)$ acts at the center of gravity along the z-axis in the ground coordinate system, so it captures the effects of pitch and roll angles in the body co-ordinate system. The nose gear steering angle is denoted by $\delta$; no other wheels are used to steer the aircraft. Orthogonal tire forces $\left(F_{x N, R, L}, F_{y N, R, L}\right.$, $\left.F_{z N, R, L}\right)$ are defined at the tire-ground contact patch. The aerodynamic forces $\left(F_{x A}, F_{y A}, F_{z A}\right)$ and moments $\left(M_{x A}, M_{y A}, M_{z A}\right)$ act at (or about) the aerodynamic center of the aircraft. The thrust forces $\left(F_{x T R}\right.$ and $\left.F_{y T R}\right)$ act parallel to the $\mathrm{x}$-axis of the aircraft.

A ground coordinate system is considered in order to describe the aircraft's motion on the ground. The global position of the aircraft's CG is defined in a global Cartesian frame $(X, Y, Z)$ while the attitude of the airframe is defined by Euler angles $(\psi, \theta, \varphi)$, where $\psi$ is the yaw angle, $\theta$ the pitch angle and $\varphi$ the roll angle. The aircraft's translational velocities and angular velocities in the ground coordinate system are defined as $\left(V_{x G}, V_{y G}, V_{z G}\right)$ and $\left(W_{x G}, W_{y G}, W_{z G}\right)$ respectively. Transformations from the local body coordinate system to the ground coordinate system use the standard sequence of rotations given in Philips [13]. By assuming that the pitch angle and roll angle remain relatively small, the velocities in the ground coordinate system are expressed as:

$$
\begin{aligned}
& V_{x G}=V_{x} \cos (\psi)-V_{y} \sin (\psi)+\theta V_{z} \cos (\psi)+\varphi V_{z} \sin (\psi) \\
& V_{y G}=V_{x} \sin (\psi)+V_{y} \cos (\psi)+\theta V_{z} \sin (\psi)+\varphi V_{z} \cos (\psi) \\
& V_{z G}=-\theta V_{x}+\varphi V_{y}+V_{z} \\
& W_{y G}=W_{y}-\varphi W_{z} \\
& W_{x G}=W_{x}+\theta W_{z} \\
& W_{z G}=W_{y} \varphi / \cos (\theta)+W_{z}
\end{aligned}
$$


Another six ODEs are therefore introduced to calculate aircraft's position and attitude in the global reference frame:

$$
\begin{gathered}
\dot{X}=V_{x G} \\
\dot{Y}=V_{y G} \\
\dot{Z}=V_{z G} \\
\dot{\psi}=W_{z G} \\
\dot{\theta}=W_{y G} \\
\dot{\varphi}=W_{x G}
\end{gathered}
$$

\begin{tabular}{|c|c|c|}
\hline Symbol & $\begin{array}{c}\text { Parameter } \\
\text { Dimensions relative to } C G \text { position }\end{array}$ & Value \\
\hline$l_{x N}$ & $\mathrm{x}$-distance to the nose gear & $\left(10.186+C G \div 100 \times l_{\text {mac }}\right) \mathrm{m}$ \\
\hline$l_{z N}$ & $\mathrm{z}$-distance to the nose gear & $2.932 \mathrm{~m}$ \\
\hline$l_{x R}, l_{x L}$ & $\mathrm{x}$-distance to the right/left gear & $\left(2.498-C G \div 100 \times l_{m a c}\right) \mathrm{m}$ \\
\hline$l_{y R}, l_{y L}$ & $\mathrm{y}$-distance to the right/left gear & $3.795 \mathrm{~m}$ \\
\hline$l_{z R}, l_{z L}$ & z-distance to the right/left gear & $2.932 \mathrm{~m}$ \\
\hline$l_{x A}$ & $\mathrm{x}$-distance to the aerodynamic center & $(0.25-C G \div 100) \times l_{m a c} \mathrm{~m}$ \\
\hline$l_{z A}$ & z-distance to the aerodynamic center & $0.988 \mathrm{~m}$ \\
\hline$l_{x T}$ & $\mathrm{x}$-distance to the thrust center & $(0.25-C G \div 100) \times l_{m a c} \mathrm{~m}$ \\
\hline$l_{y T R}, l_{y T L}$ & $y$-distance to the thrust center & $5.755 \mathrm{~m}$ \\
\hline$l_{z T}$ & z-distance to the thrust center & $1.229 \mathrm{~m}$ \\
\hline \multirow[t]{2}{*}{$l_{m a c}$} & Mean aerodynamic chord & $4.194 \mathrm{~m}$ \\
\hline & Wheel parameters & \\
\hline$k_{z N}$ & Stiffness coefficient of the nose tire & $1190 \mathrm{kN} / \mathrm{m}$ \\
\hline$k_{Z M}$ & Stiffness coefficient of the main tire & $2777 \mathrm{kN} / \mathrm{m}$ \\
\hline$c_{Z N}$ & Damping coefficient of the nose tire & $1000 \mathrm{Ns} / \mathrm{m}$ \\
\hline$c_{Z M}$ & Damping coefficient of the main tire & $2886 \mathrm{Ns} / \mathrm{m}$ \\
\hline$c_{r r}$ & Rolling resistance coefficient & 0.02 \\
\hline \multirow[t]{2}{*}{$R_{\text {wheel }}$} & Wheel radius of the main landing gear & $0.64 \mathrm{~m}$ \\
\hline & Moment of Inertia parameters & \\
\hline$I_{x x}$ & Moment of Inertia about $\mathrm{x}$-axis & $913200 \mathrm{~kg} \cdot \mathrm{m}^{2}$ \\
\hline$I_{y y}$ & Moment of Inertia about y-axis & $2548000 \mathrm{~kg} \cdot \mathrm{m}^{2}$ \\
\hline$I_{z z}$ & Moment of Inertia about $\mathrm{z}$-axis & $3335000 \mathrm{~kg} \cdot \mathrm{m}^{2}$ \\
\hline$I_{M G}$ & Moment of inertia of main landing gear & $30.925 \mathrm{~kg} \cdot \mathrm{m}^{2}$ \\
\hline
\end{tabular}

The position $(X, Y)$ and yaw angle $\psi$ can be used to plot the trajectory of the aircraft's CG.

Table 1 System parameters and values

\section{B. Tire model}


At low velocities, the nonlinear forces generated by the tires have the dominant effect over aerodynamic forces for ground vehicles. A realistic tire model is therefore required to capture the system's response accurately. Previous work presented a model that describes the lateral force generating capabilities of the aircraft's tires [4] - this capability is extended here to include longitudinal tire dynamics. A second subscript (following $x, y$ or z) of N, R or $\mathrm{L}$ is used here to indicate the nose, right or left landing gear local coordinate system with which the velocity or force elements are aligned.

The vertical force component on the tire is modelled as a spring and damper system:

$$
\begin{gathered}
F_{z N}=-k_{z N} \delta_{z N}+c_{z N} V_{z N} \\
F_{z R}=-k_{z R} \delta_{z R}+c_{z R} V_{z R} \\
F_{z L}=-k_{z L} \delta_{z L}+c_{z L} V_{z L}
\end{gathered}
$$

The stiffness coefficients $k_{z N, R, L}$ and damping coefficients $c_{z N, R, L}$ are listed in Table 1 . The vertical velocity of each tire $\left(V_{z N}, V_{z R}, V_{z L}\right)$ is calculated in terms of the aircraft velocities in the local body coordinate system as follows [4]:

$$
\begin{aligned}
& V_{z N}=V_{z}-l_{x N} W_{y} \\
& V_{z R}=V_{z}+l_{y R} W_{x}+l_{x R} W_{y} \\
& V_{z L}=V_{z}-l_{y L} W_{x}+l_{x L} W_{y}
\end{aligned}
$$

Making the assumption that the roll axes of each tire remains parallel to the ground at all time, vertical tire deflection $\left(\delta_{Z N, R, L}\right)$ can be expressed in terms of the aircraft's position and orientation (in the ground coordinate system) as [4]:

$$
\begin{aligned}
& \delta_{z N}=-l_{z N}-Z+l_{x N} \sin (\theta) \\
& \delta_{z R}=-l_{z R}-Z-l_{x R} \sin (\theta)-l_{y R} \sin (\varphi) \\
& \delta_{z L}=-l_{z L}-Z-l_{x L} \sin (\theta)+l_{y L} \sin (\varphi)
\end{aligned}
$$

With the vertical load on each tire defined, it is possible to calculate the lateral and longitudinal forces generated by each tire. The longitudinal force generated by each tire consists of two components: rolling resistance; force caused by slip ratio (in the case of braking). Rolling resistance occurs due to hysteresis in the material of the tire, and is the primary motion resistance force at low speeds. This hysteresis in the tire causes the pressure in the leading half of the contact patch to be higher than that in the trailing half, resulting in the generation of a horizontal force 
(rolling resistance) to balance the moments about the roll axis of the tire. In the aircraft model, rolling resistance is approximated by:

$$
F_{r r N, R, L}=c_{r r} F_{z N, R, L} \cos \left(\alpha_{N, R, L}\right)
$$

Here, $\mathrm{Crr}$ is the rolling resistance coefficient (specified in Table 1). The lateral slip angle $\left(\alpha_{*}\right)$ specifies the component of the rolling resistance force that opposes the aircraft's motion. It is defined for each tire as:

$$
\alpha_{N, R, L}=\arctan \left(\frac{V_{y N, R, L}}{V_{x N, R, L}}\right)
$$

The lateral force on the nose-gear $F_{y N}$ is a nonlinear function of the slip angle $\alpha_{N}$ and can be represented by [4]:

$$
\begin{gathered}
F_{y N}=F_{y \operatorname{maxN}} \frac{2 \alpha_{o p t N} \alpha_{N}}{\alpha_{o p t N}^{2}+\alpha_{N}^{2}} \\
F_{y \operatorname{maxN}}=-3.53 \times 10^{-6} F_{z N}^{2}+8.83 \times 10^{-1} F_{z N} \\
\alpha_{o p t N}=6.14 \times 10^{-11} F_{z N}^{2}+4.89 \times 10^{-7} F_{z N}+0.24
\end{gathered}
$$

where $\alpha_{o p t N}$ is the optimal slip-angle where the maximum lateral force $F_{y \operatorname{maxN}}$ occurs.

As the study in this paper considers braking on the main-gears, a combined-slip tire model developed by Milliken [14] is used to determine both longitudinal and lateral tire forces, which each depend on the total slip (lateral slip-angle and longitudinal slip-ratio) that the tire experiences at any given point in time. When brake is applied on the main-gear, it will firstly reduce the wheel's angular velocity and develop a difference between the contact-patch speed and translational speed. This difference, expressed as a percentage, is called slip-ratio:

$$
S_{R, L}=\frac{\Omega_{R, L} R_{w h e e l}-V_{x R, L}}{V_{x R, L}}
$$

To combine the slip-angle and slip-ratio, they need to be normalized first:

$$
\begin{gathered}
\bar{\alpha}_{R, L}=\frac{C_{y R, L} \alpha_{R, L}}{\mu_{y R, L} F_{z R, L}} \\
\bar{S}_{R, L}=\frac{C_{x R, L} S_{R, L}}{\mu_{x R, L} F_{z R, L}}
\end{gathered}
$$

The combined-slip $k_{R, L}$ can then be defined as:

$$
k_{R, L}=\sqrt{\bar{S}_{R, L}^{2}+\bar{\alpha}_{R, L}^{2}}
$$

According to the model of nose-gear lateral force given by Eqn.32, the resultant (normalized) friction force $F_{r R, L}$ can be represented in a similar way, as a function of the combined-slip $k_{R, L}$ : 


$$
F_{r R, L}=\frac{2 k_{o p t R, L} k_{R, L}}{k_{o p t R, L}^{2}+k_{R, L}^{2}}
$$

The parameter $k_{o p t R, L}$ is a quadratic function of the vertical load on the tire given by:

$$
k_{\text {opt }, L}=1.34 \times 10^{-10} F_{z R, L}^{2}+1.06 \times 10^{-5} F_{z R, L}+6.72
$$

By decomposing the normalized resultant force $F_{r R, L}$, we can find the lateral and longitudinal components $\bar{F}_{y R, L}$ and $\bar{F}_{x R, L}$ from their relationship given by:

$$
\begin{aligned}
& F_{r R, L}=\sqrt{{\overline{F_{y R, L}}}^{2}+\bar{F}_{x R, L}^{2}} \\
& \bar{F}_{y R, L}=\frac{\eta_{R, L}\left(k_{R, L}\right) \bar{\alpha}_{R, L}}{\bar{S}_{R, L}} \bar{F}_{x R, L}
\end{aligned}
$$

Here $\eta_{R, L}\left(k_{R, L}\right)$ is a function of combined-slip $k_{R, L}$ that is used to define the above equation for both small and large slip angles and slip ratios, and takes the form:

$$
\eta_{R, L}\left(k_{N, R, L}\right)=\left\{\begin{array}{cl}
1 / 2\left[1+\eta_{0 R, L}\right]-1 / 2\left[1-\eta_{0 R, L}\right] \cos \left(k_{R, L}^{k_{2}}\right), & \left|k_{R, L}\right| \leq 2 \pi \\
1 \quad, & \left|k_{R, L}\right|>2 \pi
\end{array}\right.
$$

Here, $\eta_{0 R, L}$ is computed based on the longitudinal stiffness $C_{x R, L}$, cornering stiffness $C_{y R, L}$, longitudinal friction coefficient $\mu_{x R, L}$, and lateral friction coefficient $\mu_{y R, L}$ :

$$
\eta_{0 R, L}=\frac{C_{y R, L} \mu_{x R, L}}{C_{x R, L} \mu_{y R, L}}
$$

From Eqn.41, by substituting $\bar{F}_{y R, L}$ with $\bar{F}_{x R, L}$ using Eqn.42, the normalized longitudinal and lateral forces can be derived as:

$$
\begin{aligned}
& \bar{F}_{y R, L}=\eta_{R, L} F_{r R, L} \frac{\bar{\alpha}_{R, L}}{\sqrt{{\overline{S_{R, L}}}^{2}+\eta_{R, L}^{2} \bar{\alpha}_{R, L}^{2}}} \\
& \bar{F}_{x R, L}=F_{r R, L} \frac{\bar{S}_{R, L}}{\sqrt{{\overline{S_{R, L}}}^{2}+\eta_{R, L}^{2} \bar{\alpha}_{R, L}^{2}}}
\end{aligned}
$$

Finally, since the longitudinal and lateral tire friction forces are normalized with respect to the maximum friction force, the actual forces are given by:

$$
\begin{aligned}
& F_{y R, L}=F_{y \max , L} \bar{F}_{y R, L} \\
& F_{x R, L}=F_{x \max , L} \bar{F}_{x R, L}
\end{aligned}
$$


The parameters $F_{x \max , L}$ and $F_{y \max , L}$ are the maximum force that can be generated by the tire longitudinally and laterally. It is assumed that longitudinal and lateral maximum forces are equal and can be obtained from the equations:

$$
\begin{aligned}
& F_{y \max , L}=-7.39 \times 10^{-7} F_{z R, L}^{2}+5.11 \times F_{z R, L} \\
& F_{x \max , L}=F_{y \max , L}
\end{aligned}
$$

\section{Aerodynamic model}

As aerodynamic forces are proportional to the square of the incoming air velocity, they provide nonlinearity within the aircraft model in addition to the tire forces. The aircraft's aerodynamic coefficients also depend nonlinearly on the angles that the aircraft makes with the airflow: aerodynamic slip angle $\alpha_{a c}$ and attack angle $\sigma$. By invoking the assumption that the aircraft operates in still air (i.e. neglecting wind effects), and that the angle of attack remains constant during ground manoeuvres, the aerodynamic slip angle can be defined in the same way as the tire model:

$$
\alpha_{a c}=\operatorname{acrtan}\left(\frac{V_{y}}{V_{x}}\right)
$$

Here, $V_{x}$ and $V_{y}$ are the velocities of the aircraft $C G$ in the local body coordinate system. The aerodynamics model consists of six elements: three forces and three moments about each axis of the aircraft. It is assumed that all the force elements act at the aerodynamic center of the aircraft, which is defined at a position $25 \%$ along the mean aerodynamic chord from its leading edge. The six force elements are modelled as follows:

$$
\begin{aligned}
& F_{x A}=\frac{1}{2} \rho|V|^{2} S_{w} C_{x}\left(\alpha_{a c}\right) \\
& F_{y A}=\frac{1}{2} \rho|V|^{2} S_{w} C_{y}\left(\alpha_{a c}\right) \\
& F_{z A}=\frac{1}{2} \rho|V|^{2} S_{w} C_{z}\left(\alpha_{a c}\right) \\
& M_{x A}=\frac{1}{2} \rho|V|^{2} S_{w} l_{m a c} C_{l}\left(\alpha_{a c}\right) \\
& M_{y A}=\frac{1}{2} \rho|V|^{2} S_{w} l_{m a c} C_{m}\left(\alpha_{a c}\right) \\
& M_{z A}=\frac{1}{2} \rho|V|^{2} S_{w} l_{m a c} C_{n}\left(\alpha_{a c}\right)
\end{aligned}
$$

Here, $|V|$ is the aircraft's resultant velocity, and the parameters $\rho, S_{w}, l_{m a c}$ are listed in Table 1 . The aerodynamic coefficients $C_{x}, C_{y}, C_{z}, C_{l}, C_{m}, C_{n}$ are nonlinear functions of aerodynamic slip $\alpha_{a c}$. The relationship between 
aerodynamic coefficients and aerodynamic slip are obtained from the SIMMECHANICS model developed by the GARTEUR Group [18].

\section{Generalized Optimal Control}

The Generalized Optimal Control algorithm (GOC) [8][9] is used in this study to develop optimal control strategies for a variety of aircraft ground manoeuvres, where optimality is defined in the form of a continuous function (which need not be quadratic). GOC aims to minimize a Hamiltonian function, expressed in terms of system states and co-states (Lagrange multipliers), to find the optimal control sequence for a particular cost function. The following subsections describe the general formulation, and present a validation test case for the specific implementation of GOC in this paper with the aircraft model.

\section{A. General Formulation}

The GOC algorithm is based on a gradient descent implementation of Pontryagin's Maximum Principle [19] [20] for application in dynamical systems. The cost function can take any (smooth) mathematical form, but typically consists of a continuous dynamic component $L[x(t), u(t)]$ plus a residual cost $L_{T}[x(T)]$ associated with final states:

$$
J=L_{T}[x(T)]+\int_{0}^{T} L[x(t), u(t)] d t
$$

A Hamiltonian function is defined in terms of the system states $x(t)$ and co-states $p(t)$ :

$$
H=L[x(t), u(t)]+p^{T}(t) g[x(t), u(t)]
$$

The function $g$ defined from the dynamical system's ODEs written in the form:

$$
\dot{x}=g[x(t), u(t)]
$$

The co-states are obtained using the following differential equations:

$$
\begin{aligned}
& \dot{p}^{T}(t)=-\frac{\partial H}{\partial x}=-\frac{\partial L}{\partial x}-p^{T} \frac{\partial g}{\partial x} \\
& p^{T}(T)=\frac{\partial L_{T}}{\partial x(T)}
\end{aligned}
$$


Optimal control sequences are found at the minimum of the Hamiltonian function with respect to the control variables:

$$
\frac{\partial H}{\partial u}=0
$$

The equations above are all solved for $t \in[0, T]$ with two initial conditions forming a two-point boundary value problem: fixed initial states $x(0)$ and fixed final co-states $p(T)$.

The optimal solution is identified via a discrete sequence of controls, with each control element held constant for an equal time interval $\Delta t[8][9][20]$. For each control time period, the cost gradient is obtained directly from the Hamiltonian as:

$$
\frac{\partial J}{\partial u_{i}}=\int_{t_{i-1}}^{t_{i}} \frac{\partial H}{\partial u_{i}} d t
$$

A gradient-based iterative optimization can then be utilized to determine the optimal control sequence - the algorithm is depicted in Fig 3.1, with the 4 steps outlined below:

Step 1: The dynamical system is evaluated (for the current control sequence, initial guess can be zero) from the initial condition $x(0)$ using Eqn.60. The continuous cost component of Eqn.58 is integrated simultaneously.

Step 2: The final state $x(T)$ is used to evaluate the residual cost $L_{T}[x(T)]$ and the final co-state $p[T]$ using Eqn.62

Step 3: The co-state system calculates the integral of the co-states via $\partial \mathrm{H} \partial \mathrm{x}$ in reverse-time from the final co-state $p[T]$.

Step 4: Cost gradients are used to update the control sequence by a line search optimization along the steepest descent direction to minimize the cost function.

Steps 1-4 are repeatedly executed until suitable convergence is achieved.

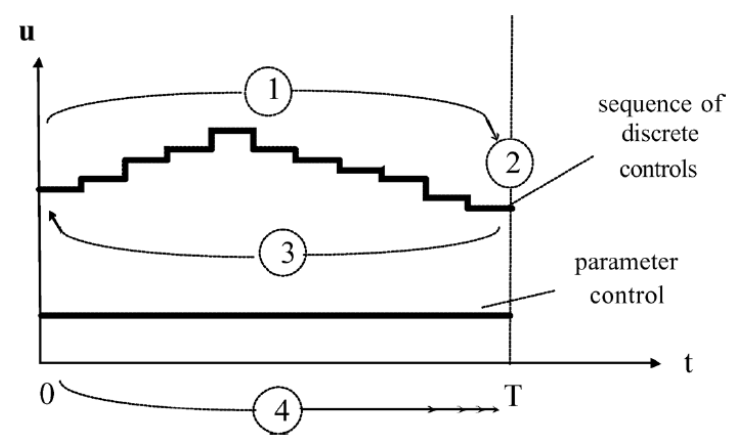

Figure 3.1 Summary of the GOC algorithm. 


\section{B. GOC Implementation Test: Maximum Deceleration Rate}

The specific implementation of GOC with the aircraft ground dynamics model, as used throughout this paper, is validated through application to a test case with known results: a maximum straight-line deceleration case. A $4^{\text {th }}$ order Runge-Kutta method is used to perform the time integration of the states and co-states, with a constant timestep used throughout the simulation. State errors are monitored in order to set the time step to ensure reasonable accuracy. A zero-order-hold control input is applied for some control timestep $\Delta \mathrm{t}$, not necessarily the same as the numerical integration timestep. The length of control step $(\Delta \mathrm{t})$ affects the convergence speed of the optimization, and also the fineness of the control sequence. To achieve a fast and accurate convergence, a coarse resolution is specified initially; this resolution is improved over several concurrent GOC runs to obtain an appropriate level of continuity in the final control sequence.

In this straight line braking scenario, GOC is used to identify the braking required to minimize the total distance travelled during a simulation. The aircraft starts in an equilibrium condition, with a forward speed of $40 \mathrm{~m} / \mathrm{s}$ and heading in the global $\mathrm{X}$ direction. The simulation time span (in seconds) is specified as $t \in[0,10]$, with a numerical time step of $5 \mathrm{~ms}$ (chosen as it is sufficiently small for this dynamical system). To ensure the aircraft remains in a straight line, the continuous cost function is defined as a track cost $L_{\text {track }}$. For this case, it is a quadratic function with respect to the aircraft's deviation from the global $\mathrm{X}$ axis:

$$
L_{\text {track }}=\int_{0}^{T} Y^{2} d t
$$

In addition to this continuous cost, a final-state cost is defined as the total distance travelled, i.e. the final distance to the starting point $(-1000,0)$ :

$$
L_{\text {final }}=(X+1000)^{2}+Y^{2}
$$

The total cost is comprised of the continuous track cost plus the final cost:

$$
L_{\text {total }}=L_{\text {track }}+L_{\text {final }}
$$

The final optimal solution is shown in Fig 3.2. This optimal solution, obtained after 2200 iterations, is judged to be optimal based on the magnitude of all cost gradients at each point in time. The cost gradients obtained at this optimal iteration are shown in Fig 3.2(f): they have been reduced by several orders of magnitude compared with the initial cost gradients, showing that additional iterations beyond this point will not provide significant reductions in total cost. The optimal control sequence, in this case braking torque, is shown in Fig 3.2(a). An initial brake spike is 
used to slow the wheel speed down rapidly at the start of the simulation, however the brake torque is then modulated to keep each wheel rolling at its optimum total slip.

The oscillations in brake torque that occur during the first 4 seconds are a response to the changing dynamic load on the main landing gears, observable through comparison of Fig 3.2(a) and Fig 3.2(e). Since the tire's peak force is a function of its vertical load, a greater load on the tire means more brake torque can be applied before it saturates. Fig 3.2(e) shows how the load on the nose gear and the main gear varies throughout the simulation. Initially, there is a small weight transfer from the main gears to the nose gear due to the initial deceleration. This results in a period of transient behaviour as the longitudinal weight transfer settles to a steady value, with a corresponding reduction in brake torque during this transient period shown in Figure 3.2(a). Over the second half of the simulation, the loads on main gear and nose gear increase together, because the aircraft's lift decreases as it slows down.

Fig 3.2(b) shows the main-gears wheel contact-patch speeds and their translational speeds. The difference between them is the longitudinal slip ratio. Applying a brake torque causes the wheel's angular velocity to reduce. Since the wheel's contact-patch speed becomes lower than the translational speed, a negative slip ratio occurs. This generates a negative friction force on the main gears, which causes the aircraft to decelerate. The gradient of the dashed curve (the wheel's translational velocity) shows that the aircraft experiences a constant deceleration rate (around $3 \mathrm{~m} / \mathrm{s}^{2}$ ). This suggests that optimal braking has been achieved throughout the simulation for the aircraft's maximum deceleration rate.

Optimality of the results is validated by considering tire behaviour. For pure braking in a straight line, the total slip consists of longitudinal slip ratio only - lateral slip remains zero throughout. The maximum deceleration should therefore occur when the total slip equals the optimal slip. If the tire experiences a slip greater to or less than this value, it will generate less force than it has the capacity to do. The results from GOC, shown in Fig 3.2(c), show this is the case here. A very small period of over-slip at the beginning is caused by the initial brake spike (required to remove wheel momentum as fast as possible). Fig 3.2(d) shows the normalized tire forces (w.r.t. the maximum force) have been perfectly controlled, as the forces remain at the value of ' 1 ' for both the left and right gears. Note that no braking force is applied to the nose-gear.

This simple test case shows that the implementation of GOC used in this work is able to obtain an optimal sequence for a control input. The wheel speeds are controlled such that they remain just below saturation, in order to 
achieve the maximum deceleration rate for the whole vehicle. The next section considers results from GOC for the more complicated case of a high-speed runway exit manoeuvre.

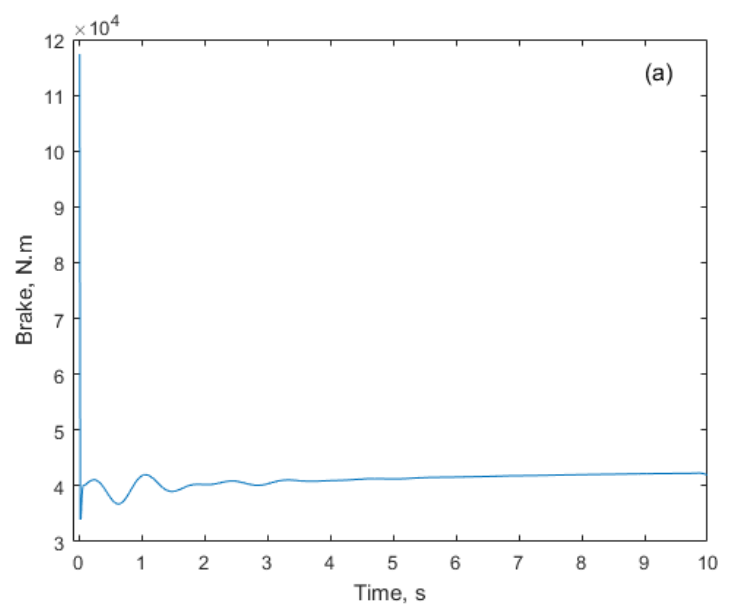

Fig 3.2(a) Optimal brake torque profile.
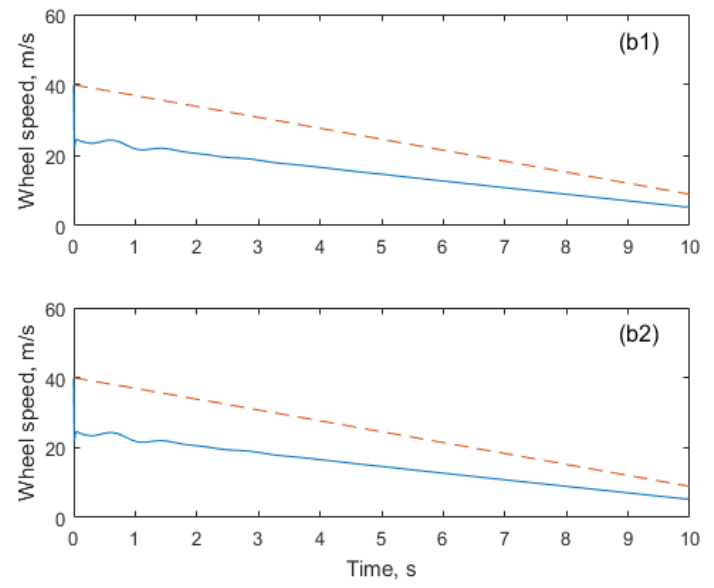

Fig 3.2(b) Wheel contact-patch speed (solid line) and translational speed (dashed line) for the right (b1) and left (b2) gear. 

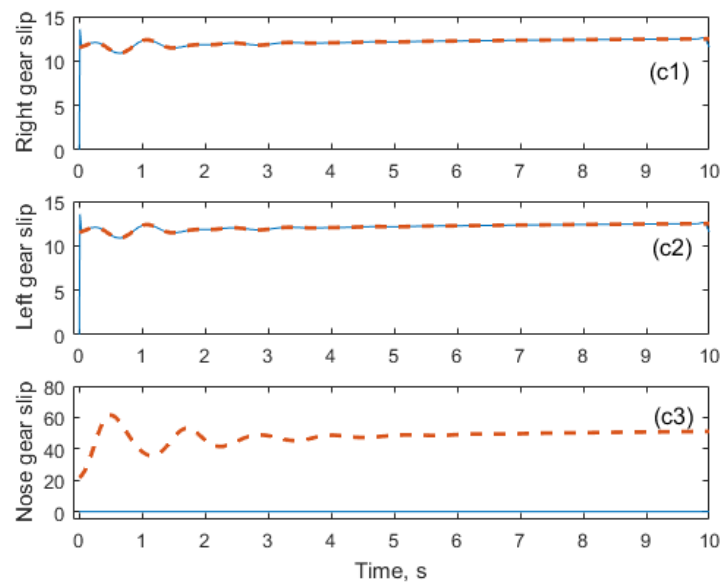

Fig 3.2(c) Optimal slip (dashed line) and actual slip (solid line) for the right (c1), left (c2), and nose (c3) gear.
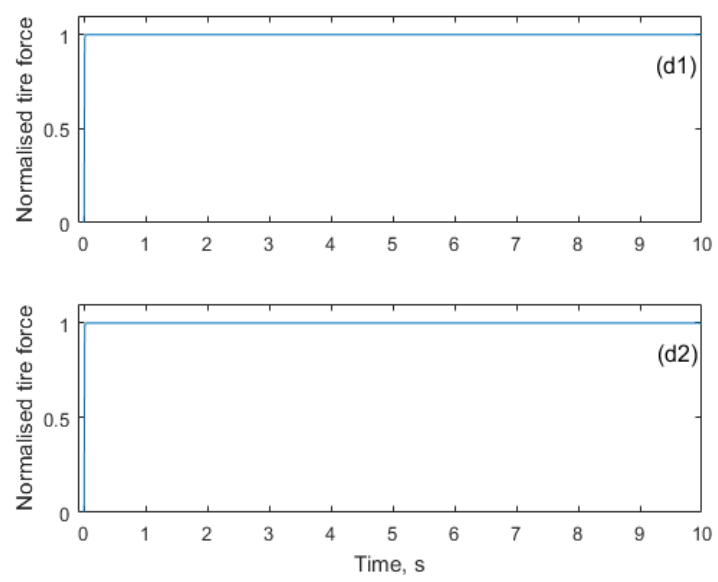

Fig 3.2(d) Normalized friction force for the right (d1) and left (d2) gear.

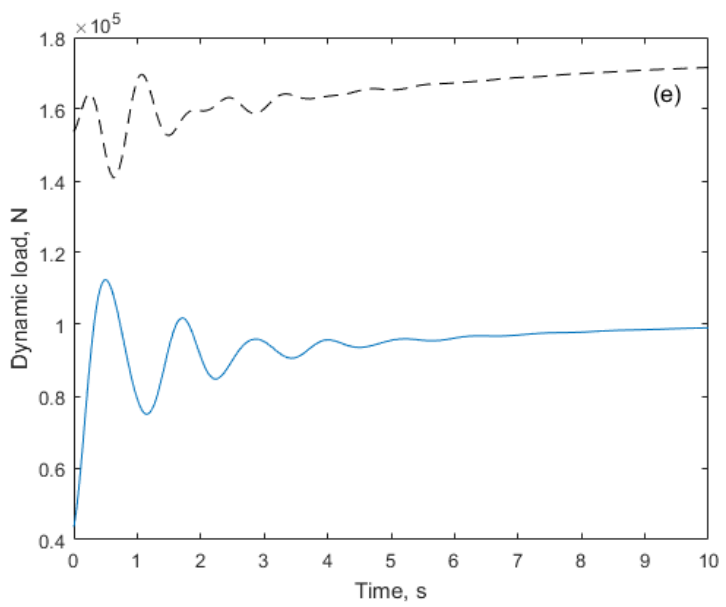

Fig 3.2(e) Dynamic load on the nose gear (solid line) and main gears (dashed line). 


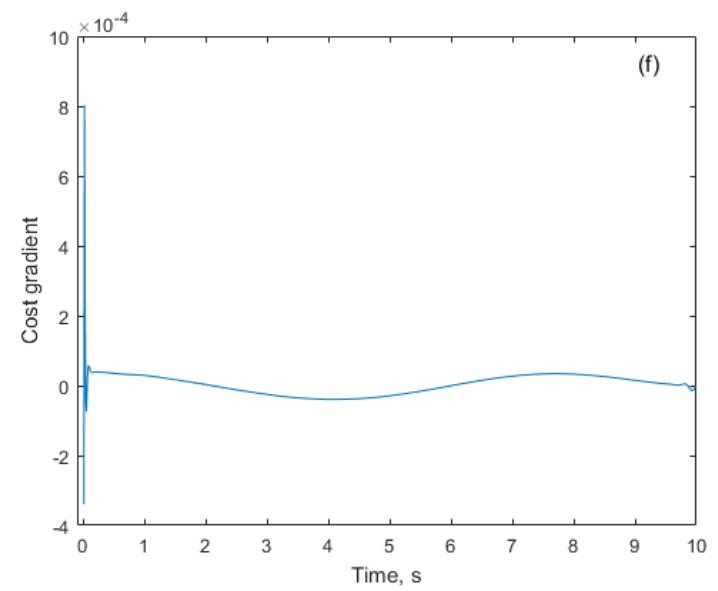

Fig 3.2(f) Cost gradient with respect to brake input.

\section{Optimization of High-speed Runway Exit}

In this section, GOC is used to find optimal control sequences for a high-speed runway exit manoeuvre. Since GOC works on fixed time interval, the interval is set as 20 seconds which is sufficiently long for the aircraft to settle down. The geometry of the runway exit considered here is shown in Fig 4.1(a). The black lines represent a safe boundary for the aircraft's CG to operate within. The track cost is defined as a quadratic function of the distance to the runway's centerline. Constraints on states and control inputs can be imposed via tanh function or higher order polynomial when needed. Such constraint should not be solely used as the track cost because aircraft will cut the corner which is quite dangerous in practice. Three cases are considered here, each using the same runway geometry and track cost: in case A, the optimal control sequence minimizes the distance between the aircraft's c/g and the runway centreline; in case $\mathrm{B}$, it maximizes the distance travelled by the aircraft during the 20 seconds of simulation time; in case $\mathrm{C}$, the optimal control sequence minimizes tire wear. 


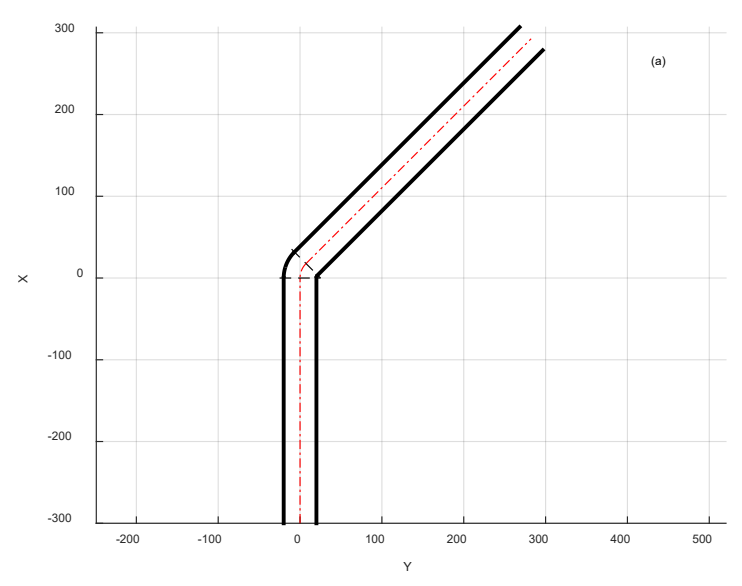

Fig 4.1(a) The 45-degree runway exit geometry in the ground $X-Y$ coordinate system.

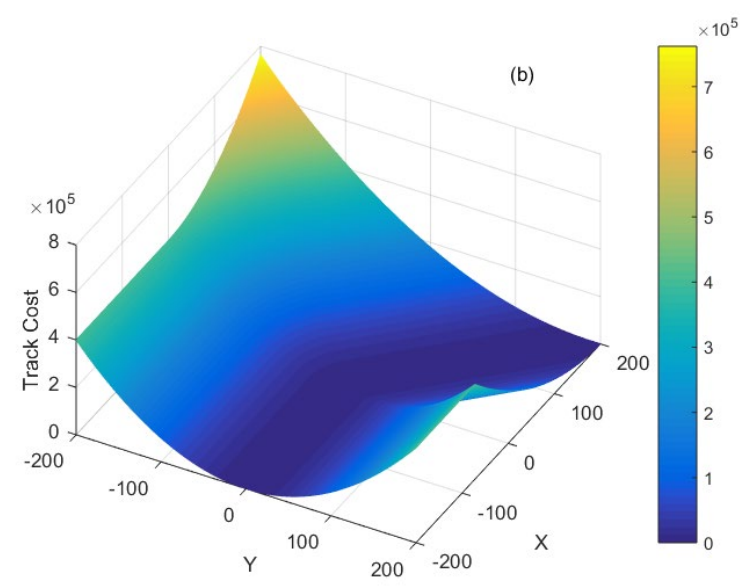

Fig 4.1(b) The track cost as a function of ground position.

\section{A: Runway exit following the centerline}

This first case uses GOC to determine a control sequence of brake, thrust and steer inputs, to minimize the distance between the aircraft's CG and the runway centerline. The initial condition is an equilibrium state with a forward speed of $40 \mathrm{~m} / \mathrm{s}$. The continuous cost function is the track cost as depicted in Fig 4.1(b). The residual cost is a function of the aircraft's yaw angle, yaw rate and lateral velocity, and is used to ensure that the aircraft finishes the simulation aligned with the road with no significant yaw rate or side-slip velocity. Hence, the overall cost function is defined as follows with coefficients $\lambda$ which are able to balance cost components; to improve convergence of the optimal solution, the $\lambda$ coefficients are set based on the residual cost and then rescaled to ensure that all the cost components are in the same order of magnitude: 


$$
\begin{aligned}
& L_{\text {final }}=\lambda_{1}(\psi-\pi / 4)^{2}+\lambda_{2} W_{z}^{2}+\lambda_{3} V_{y}^{2} \\
& L_{\text {total }}=L_{\text {track }}+L_{\text {final }}
\end{aligned}
$$

Fig 4.2(a) shows the total cost for each of the 50000 iterations. This drops dramatically within the first 1000 iterations and then settles down gradually over 49000 iterations. Since the magnitude of the cost gradients has been reduced by a factor of 10000 as shown in Fig 4.2(b), and no significant reduction of total cost can be achieved with further iterations, the cost function is judged to have converged. The spike on the cost gradient would shrink with further iterations, but it is sufficiently small (of the order $10^{-4}$ ) to be considered as converged.

The aircraft's optimal trajectory is illustrated in Fig 4.2(c), with small triangles used to represent the aircraft's position and orientation at each second of the simulation. It can be seen that the aircraft remains close to the centerline throughout the simulation, although it does not follow it exactly. The aircraft initially steers to the left when approaching the right-hand exit, in order to maximize the turning radius without deviating from the path centerline too much in the corner (and hence incurring a high cost). Fig 4.3(a). shows the optimal control inputs for steering, thrust and braking. The negative steering angle at the beginning of the simulation turns the aircraft to the left, however the aircraft's large yaw inertia means that the aircraft continues to move straight along the runway centerline for the first 3 seconds of the simulation. During the first few seconds, the brakes are applied (along with some reverse thrust) to reduce the aircraft's speed, as the initial forward speed of $40 \mathrm{~m} / \mathrm{s}$ is too fast to make a 45 degree turn. Fig 4.3(b) shows the wheel's contact-patch speed and translational speed of the right and left gear, highlighting that all wheels' translational speed drops dramatically from $40 \mathrm{~m} / \mathrm{s}$ to less than $20 \mathrm{~m} / \mathrm{s}$ over the first 6 seconds. It can be seen that the right (inner) gear's wheel speed drops towards zero during peak braking, and that the brake torque is released to a lower level before this gear's wheel locks. By doing this, the aircraft's dynamics are still under control and the tires can be recovered from their saturation states. 


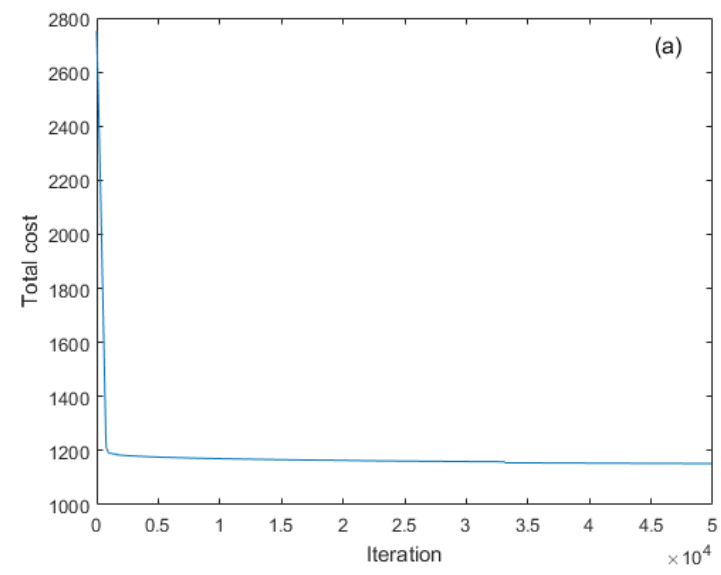

Fig 4.2(a) The total cost along the 50,000 iterations.

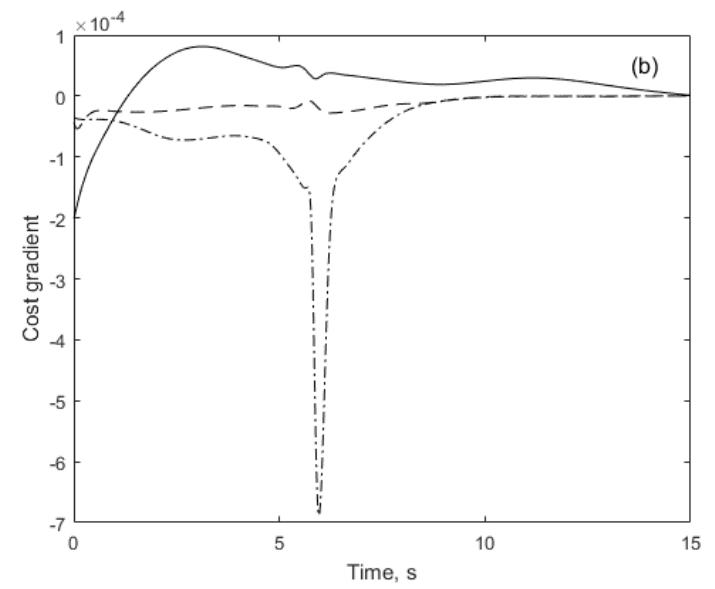

Fig 4.2(b) The final cost gradient in terms of steer (solid line), thrust (dashed line), and brake (dashdot line).

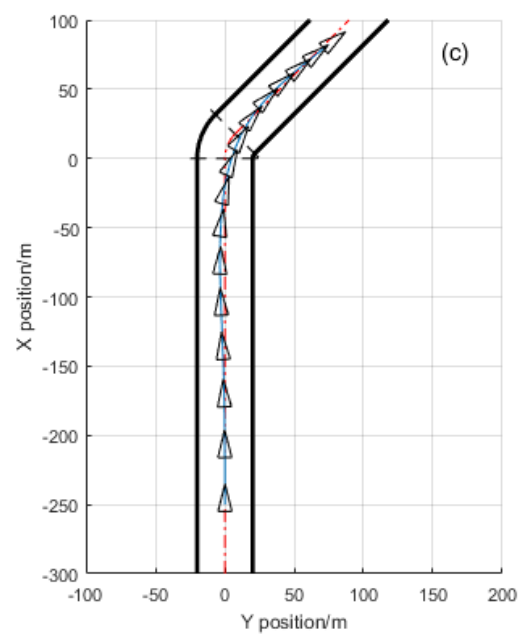

Fig 4.2(c) The optimal trajectory of the aircraft $C G$ with small triangles illustrating the aircraft position and orientation at an interval of 1 sec. 
After the 5th second, a rapid increase in braking torque causes the right gear's tire to saturate, as shown by the plot of tire slip angles in Fig 4.3(c). An over-slip for the right gear occurs, where the total slip exceeds the optimal slip and the tire is said to have saturated. This tire saturation leads to the decrease of the right gear's lateral friction force as shown in Fig 4.3(d), which in turn increases the total yaw moment around the $\mathrm{z}$ axis, allowing the aircraft to make the turn more easily. Optimality of this part of the solution is reflected in Fig 4.3(f), which shows the normalized total friction force on the main gears (normalized with respect to the maximum force that the tire can generate). It can be seen that the normalized force on the right gear stays right at the maximum level during the turn except when it over-slips to make a quick adjustment to the yaw angle.
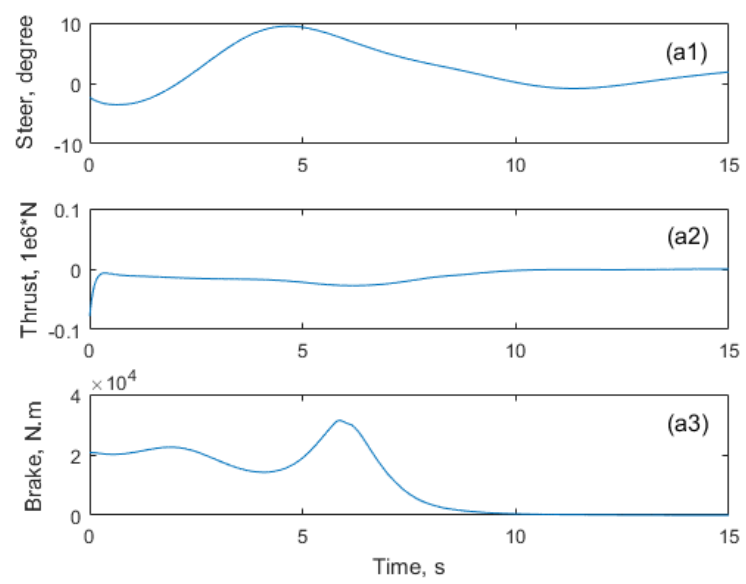

Fig 4.3(a) The optimal control input of steering (a1), thrust (a2), and braking (a3).
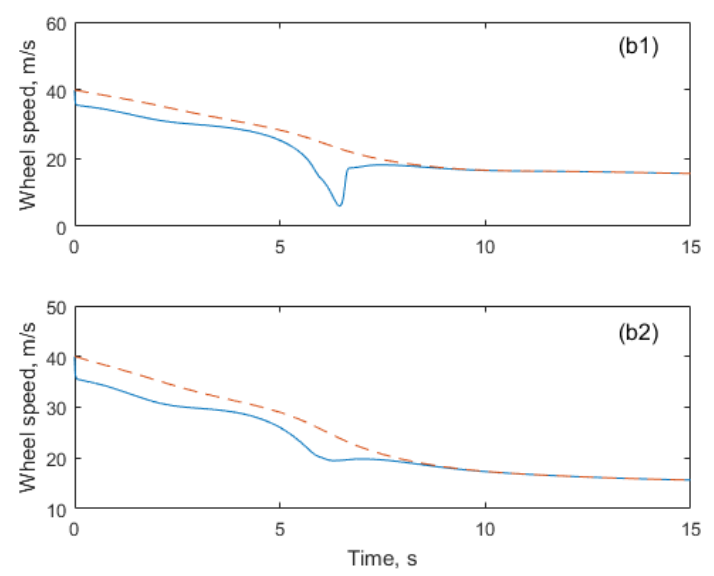

Fig 4.3(b) Wheel contact-patch speed (solid line) and translational speed (dashed line) of the right (b1) and left (b2) gear. 

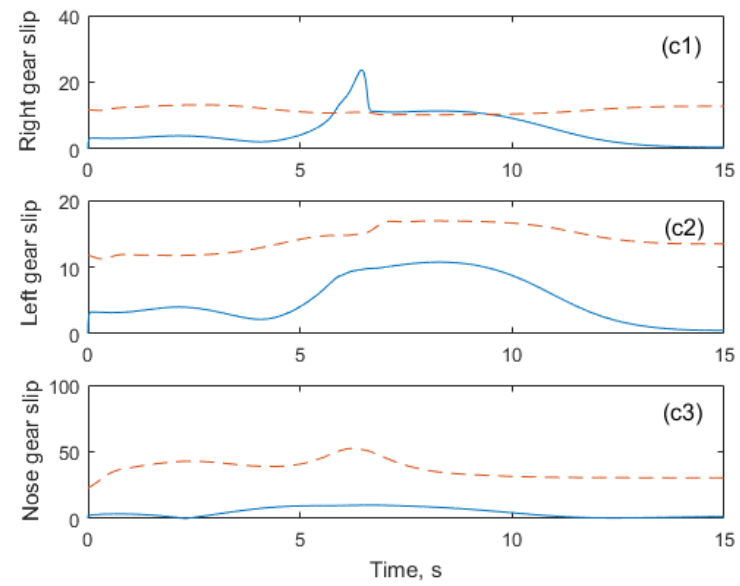

Fig 4.3(c) The optimal slip (dashed line) and the actual slip (solid line) of the right (c1), left (c2), and nose (c3) gear.
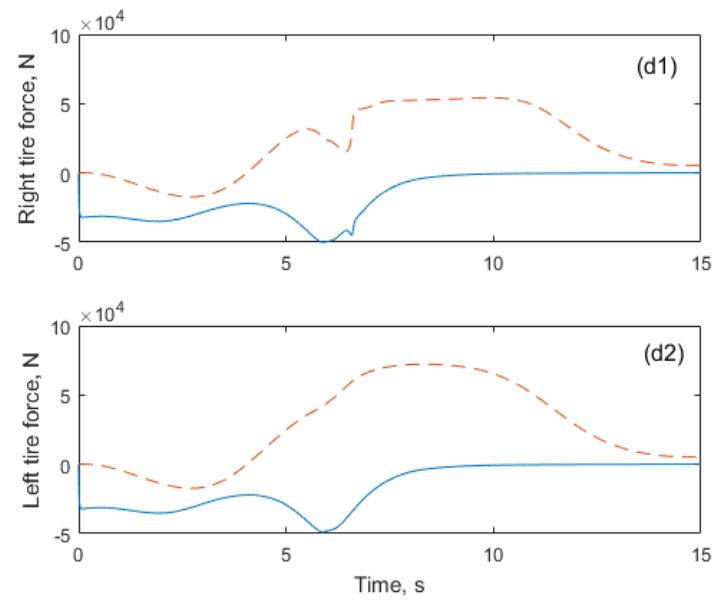

Fig 4.3(d) The longitudinal (solid line) and lateral (dashed line) force element of the total tire/ground friction force of the right (d1) and left (d2) gear.

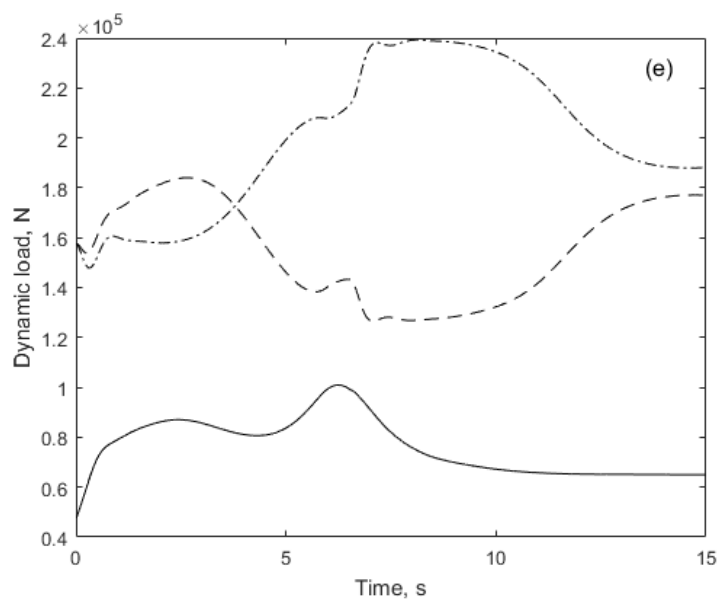

Fig 4.3(e) The dynamic load on the nose gear (solid line), right gear (dashed line), and left gear (dashdot line). 

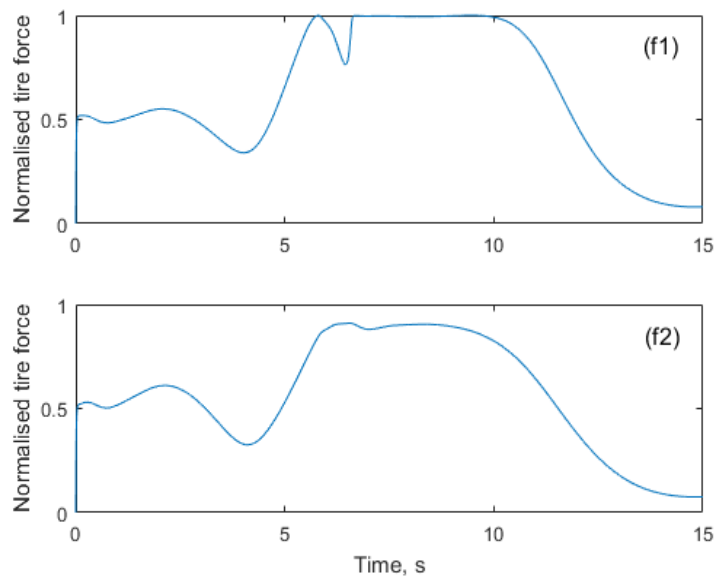

Fig 4.3(f) The normalized total tire/ground friction force of the right (f1) and left (f2) gear.

\section{B: Fast exit using brakes and steer}

This second case extends the first case by requiring that high-speed runway exit be performed as fast as possible, using steer and brake inputs (thrust are set idle in this case). A simulation time step of $10 \mathrm{~ms}$ was found to be sufficiently small for this dynamic system. To make comparison with the result in Case A, the simulation starts from the same initial condition and runs for the same period of time. The continuous cost function is the same as in Case A, however the final cost has been changed: an additional final cost is now defined with respect to the aircraft's final distance from a target point, which ensures that the control strategy maximizes distance travelled during the simulation (and hence maximizes the aircraft's runway exit speed). The final cost function used for this case is:

$$
L_{\text {final }}=\lambda_{1}(\psi-p i / 4)^{2}+\lambda_{2} W_{z}^{2}+\lambda_{3} V_{y}^{2}+\lambda_{4}\left((X-500)^{2}+\left((Y-500)^{2}\right)\right.
$$

The coefficients $\lambda_{1}, \lambda_{2}, \lambda_{3}$ remain the same as in Case A, whilst $\lambda_{4}$ is set to ensure that the cost component used to maximize distance travelled contributes approximately the same order of magnitude to the final cost as all the others. 


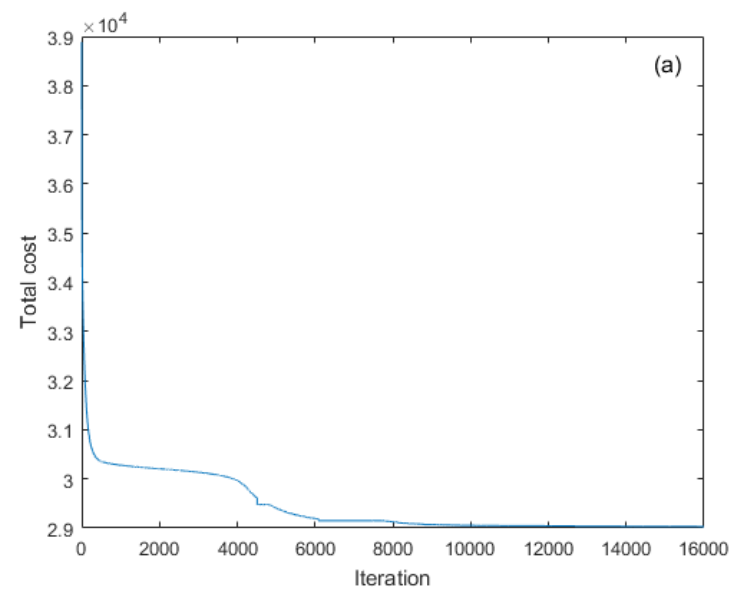

Fig 4.4(a) The total cost along the 16,000 iterations.

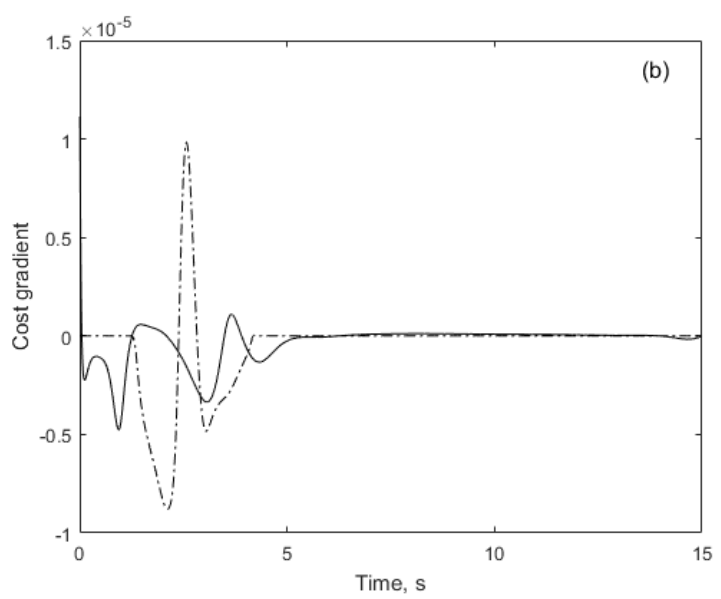

Fig 4.4(b) The final cost gradient in terms of steer (solid line) and brake (dashdot line).

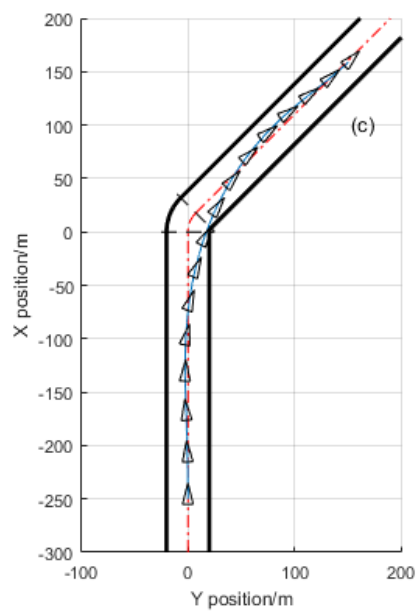

Fig 4.4(c) The optimal trajectory of the aircraft CG with small triangles illustrating the aircraft position and orientation at an interval of 1 sec. 
The cost function is judged to have converged after 16,000 iterations. The cost drops significantly in the first few hundred iterations, and then continues to drop gradually over the subsequent 9000 iterations. The magnitude of the final cost gradients, as shown in Fig 4.4(b), are of the order $10^{-5}$, so further reductions in total cost won't be achieved with additional iterations. The aircraft's final trajectory is illustrated in Fig 4.4(c), again with each small triangle showing the aircraft's position and orientation at each second of the simulation.
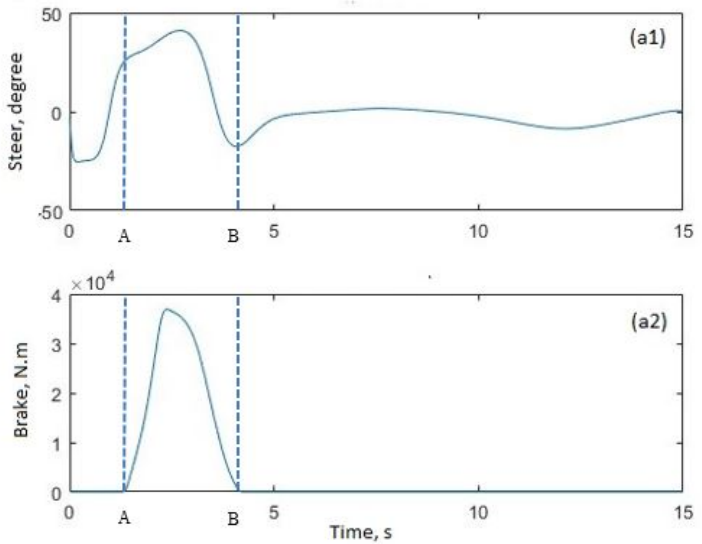

Fig 4.5(a) The optimal control input of steering and braking.
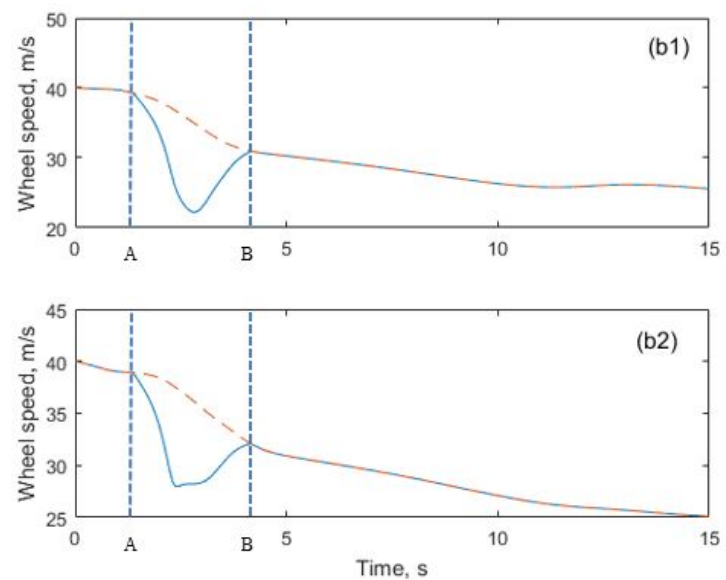

Fig 4.5(b) The wheel contact-patch speed (solid line) and the translational speed (dashed line) of the right (b1) and left (b2) gear. 

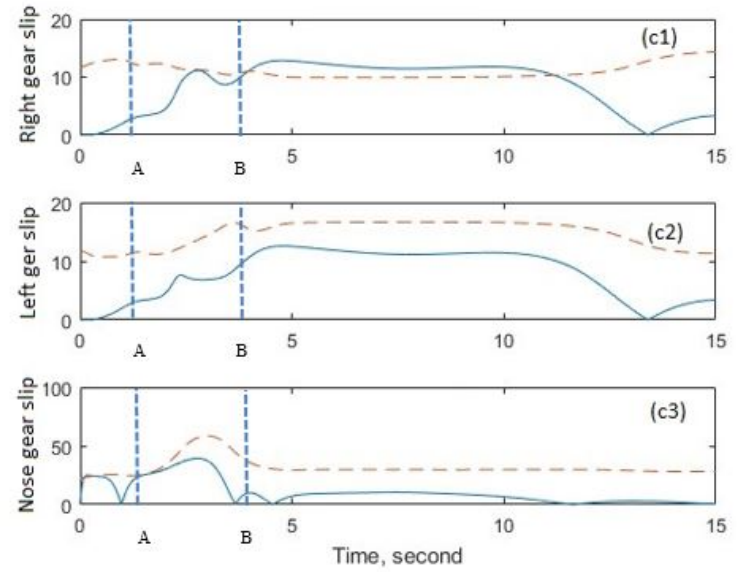

Fig 4.5(c) The optimal slip (dashed line) and the actual slip (solid line) of the nose, right and left gear.
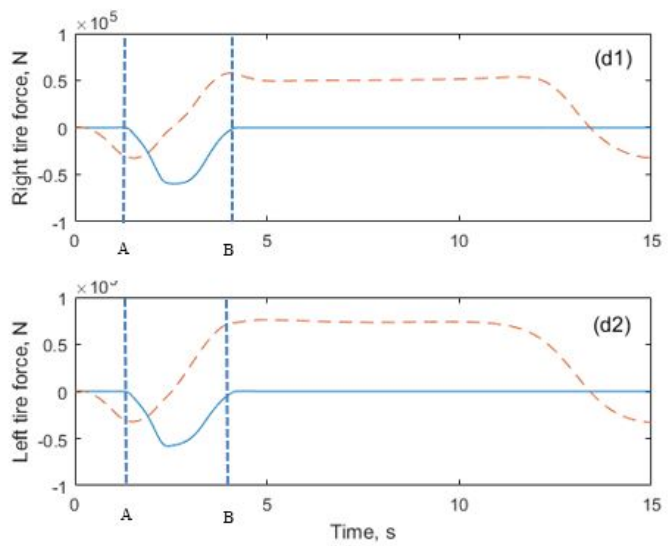

Fig 4.5(d) The longitudinal (solid line) and lateral (dashed line) force element of the total tire/ground friction force of the right (d1) and left (d2) gear.

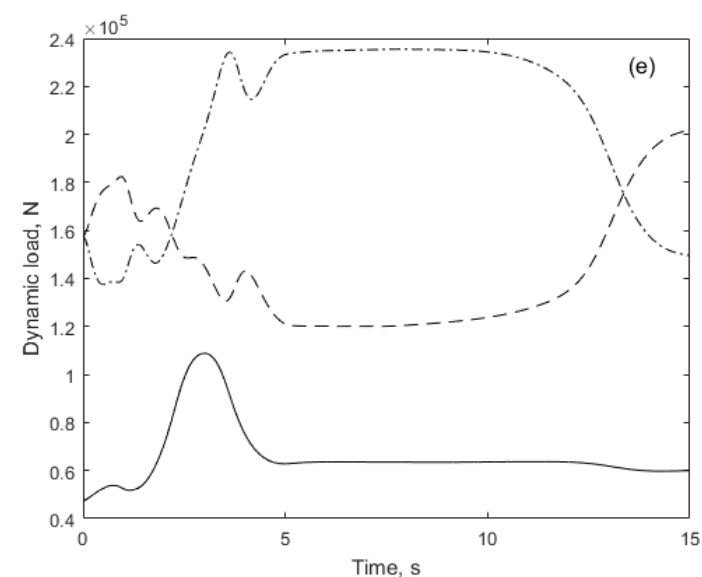

Fig 4.5(e) The dynamic load on the nose (solid line), right (dashed line) and left (dashdot line) gear. 

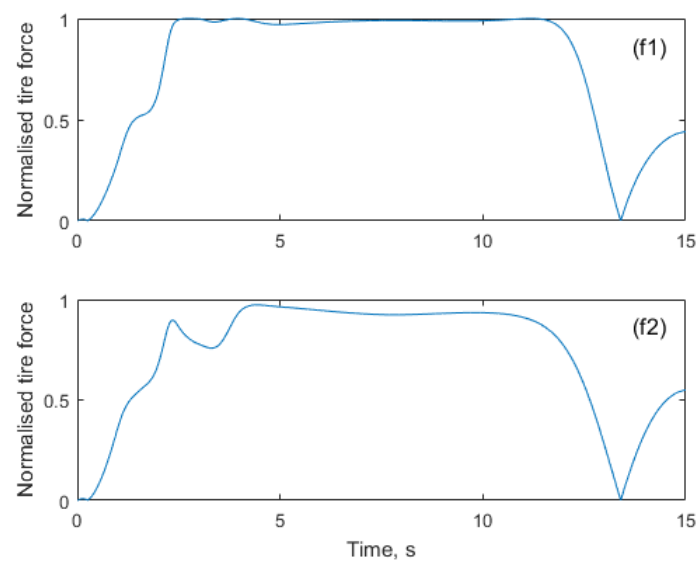

Fig 4.5(f) The normalized total tire/ground friction force of the right (f1) and left (f2) gear.

The final optimal solution is presented in Fig 4.5, with the optimal steering and braking control sequences shown in Fig 4.5(a). As for the first case, the aircraft initially steers to the left to obtain a larger turning radius, and then to the right to negotiate the coner. It also takes a couple of seconds for the steer input to build up the aircraft's yaw angle due to the aircraft's large yaw moment of inertia. Unlike the initial case where the aircraft just aims to follow the centreline, the right turn cuts more of the corner: this is a familiar tactic used by racing car drivers, as it increases the radius of the turn and hence increases the maximum speed that can be achieved by a given vehicle in the corner. The brakes are also used differently here from the first case: no brakes are applied until the aircraft is at point ' $\mathrm{A}$ ' (Fig 4.5(a)); the aircraft brakes from point 'A' to point 'B', whilst it is still steering hard right (in anticipation of the corner); the brake is completely cut off at point ' $\mathrm{B}$ ' to achieve the maximum lateral slip angle, just as the aircraft starts to move to the right of the centerline, cutting the corner. The combined use of braking and steering between points A and B is an aircraft-specific tactic, as unlike racing cars, aircrafts have huge yaw moment inertia. Since the main-gear's slip angle takes time to build up when a steering angle is actuated by the nose-gear, the action of steering doesn't immediately affect its capacity to brake by increasing the total slip experienced. In fact, Fig 4.5(c) shows that the action of braking increases the nose wheel's optimal slip: the solid curve illustrates the total slip and the dashed curve illustrates the optimal slip where the maximum force occurs. The GOC algorithm balances steer and brake inputs so that the total slip on all the three gears achieves their optimal slip value at some point during the manoeuvre, meaning maximum tire forces can be generated. This is shown in Fig 4.5(f), where the normalized tire 
force reaches a value of 1 to indicate that the tire is operating at its maximum capacity. The weight transfer from right gear to left gear, as depicted in Fig 4.5(e), means that the right gear is allowed to saturate slightly during the right turn, so that the left gear can generate more lateral force (hence maximizing the total lateral force).

\section{C: Minimum tire wear}

The final case considers minimization of tire wear as an additional continuous cost, based on the common assumption that tire wear is proportional to the frictional work performed by the tire [22][23]. Hence the tire wear cost function is given by:

$$
L_{\text {tire }}=\int_{0}^{T}\left(\left|F_{x N, R, L} V_{x N, R, L}\right|+\left|F_{y N, R, L} V_{y N, R, L}\right|\right) d t
$$

Adding this cost component to the cost function considered in Case B, the total cost in Case $\mathrm{C}$ is given by:

$$
L_{\text {total }}=L_{\text {track }}+L_{\text {tire }}+\lambda_{1}(\psi-\pi / 4)^{2}+\lambda_{2} W_{z}^{2}+\lambda_{3} V_{y}^{2}
$$

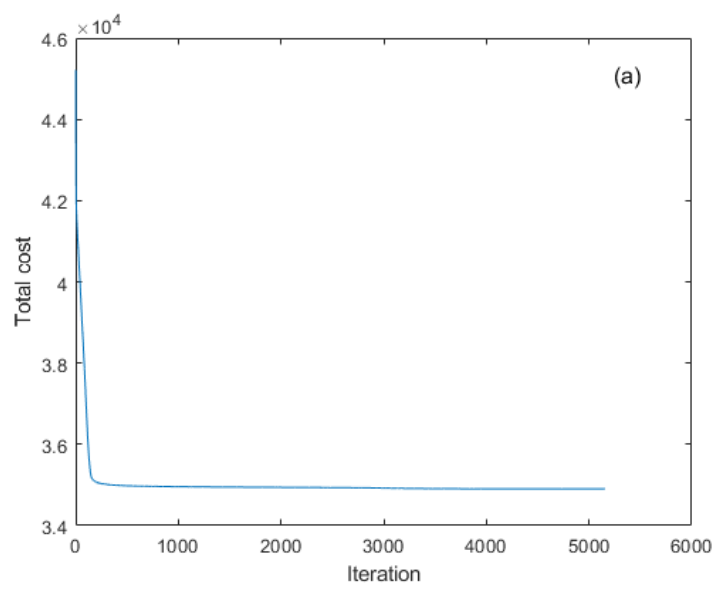

Fig 4.6(a) The total cost along with iterations. 


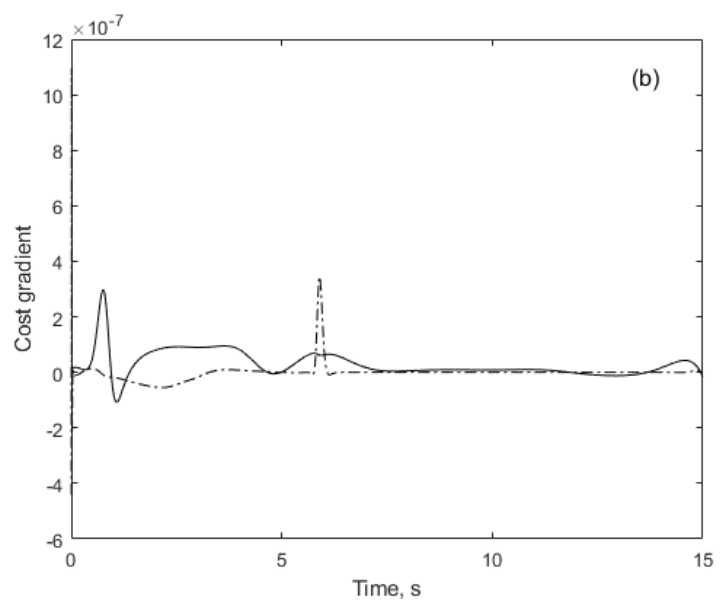

Fig 4.6(b) The final cost gradient in terms of steer, thrust and brake.

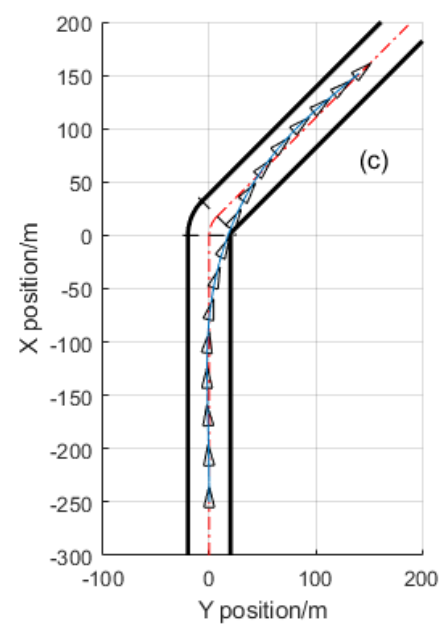

Fig 4.6 (c) shows the optimal trajectory of the aircraft CG with small triangles illustrating the aircraft position and orientation at an interval of 1 sec.

The simulation starts from the same equilibrium as used in the first two cases. The cost function converged after around 5000 iterations, as shown in Fig 4.6(a). The cost gradients of steer and brake are both of the order $10^{-7}$, as shown in Fig 4.6(b), showing that the minimum point of the cost function has been achieved. The corresponding optimal trajectory of the aircraft's CG is depicted in Fig 4.6(c), with small triangles illustrating the position and orientation of the aircraft at an interval of $1 \mathrm{sec}$.

The optimal control and the intermediate aircraft dynamics are illustrated in Fig 4.7, with optimal control sequences of steering and braking shown in Fig 4.7(a). Compared with the solution in Case B, less steering and braking is applied due to the tire wear cost. The aircraft tends to brake earlier to avoid excessive braking when approaching the exit. Consequently, the main gears' wheel speed in Fig 4.7(b) is lower than the previous case which 
in turn reflects a lower slip ratio. In Fig 4.7(c), the solid curve illustrates the total slip and the dashed curve illustrates the optimal slip where the maximum force occurs: the actual slip of all three gears is kept at a lower level throughout the manoeuvre and no over-slip occurs on any tire. The lateral and longitudinal forces of the right and left tires are shown in Fig 4.7(d); It can be seen that both force components are kept at a lower level. The corresponding normalized friction forces of the main gears in Fig 4.7(f) show more clearly that the tires are not working at their maximum capacity. Particularly, the left tire is far from its saturation point.
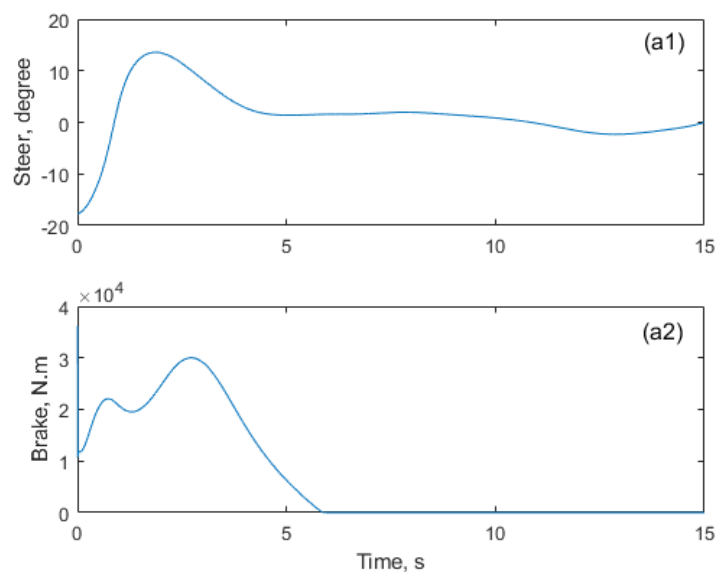

Fig 4.7(a) The optimal control input of steering and braking.
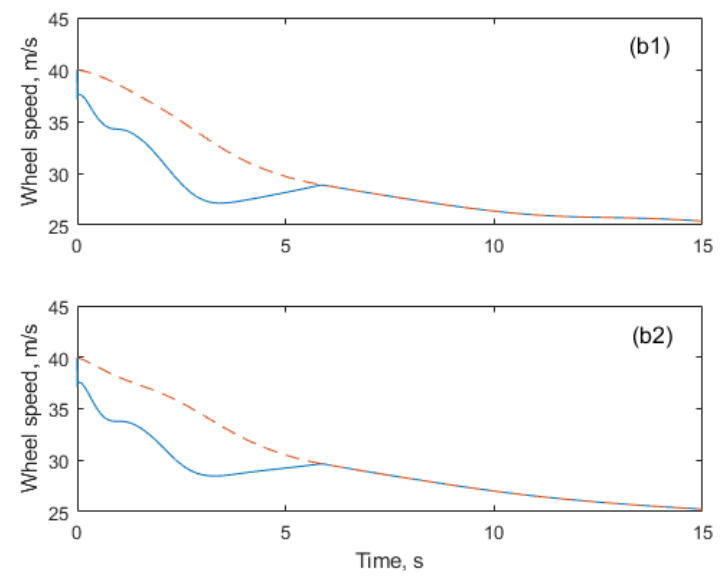

Fig 4.7(b) The wheel contact-patch speed (solid line) and translational speed (dashed line) of the right and left gear. 

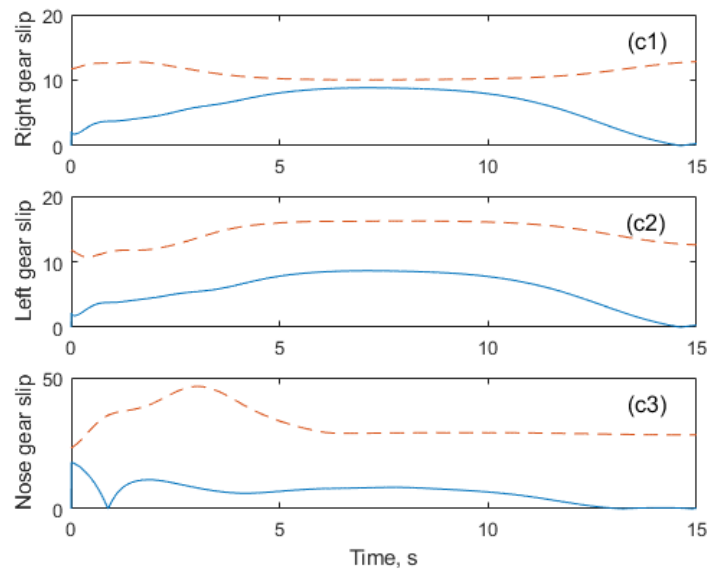

Fig 4.7(c) shows the optimal slip (dashed line) and the actual slip (solid line) of the nose, right and left gear.
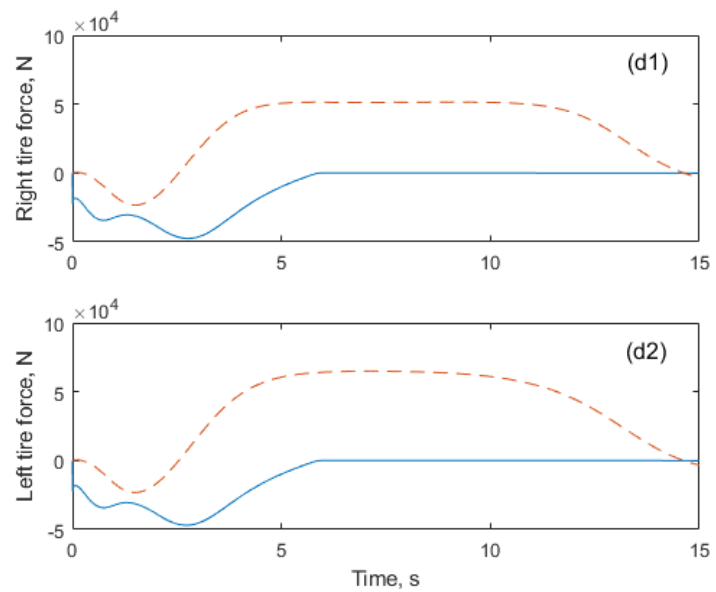

Fig 4.7(d) shows the normalized total tire/ground friction force of the right and left gear.

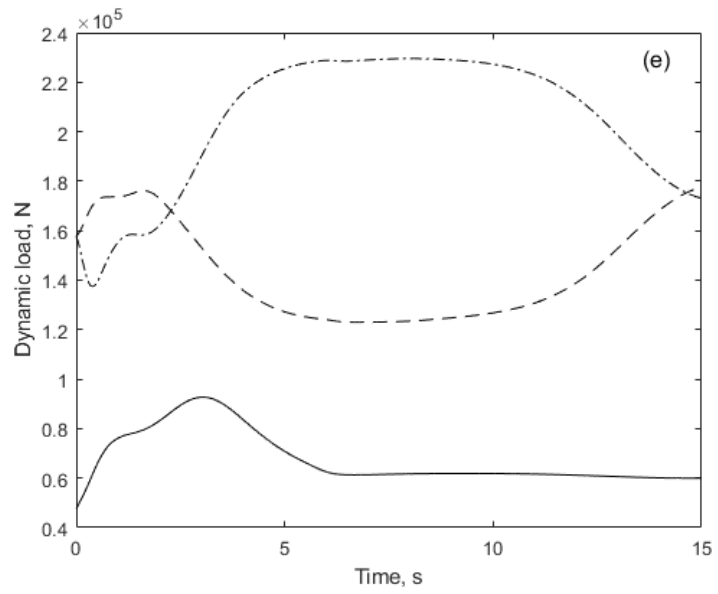

Fig 4.7(e) shows the dynamic load on the nose, right and left gear. 

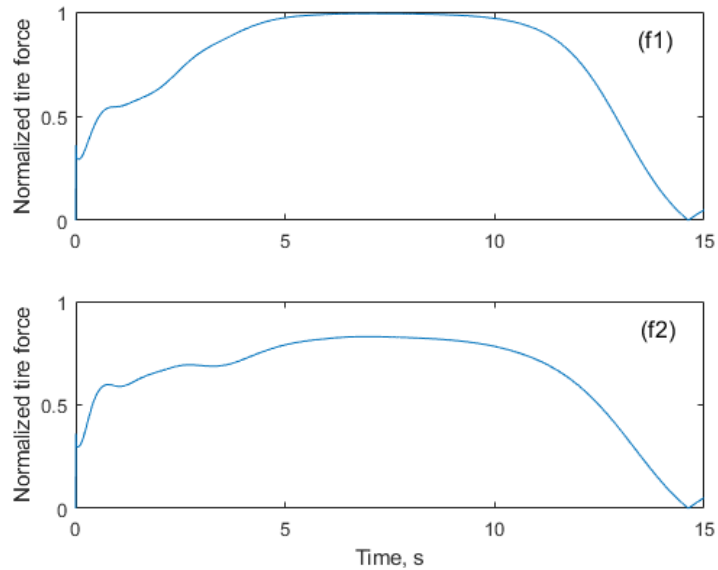

\section{Fig 4.7(f) shows the longitudinal (solid line) and lateral (dashed line) force element of the total tire/ground friction force.}

\section{Conclusion}

This paper has used a method of optimal control, known as Generalized Optimal Control, to study optimal control strategies for an aircraft undertaking a high-speed runway exit manoeuvre. The ability of GOC to define an optimal control sequence is validated with the case of a straight line maximum deceleration manoeuvre. Optimality is demonstrated because the tires on both main landing gears experience a longitudinal slip equal to their optimal slip value throughout the simulation. Three scenarios were investigated for the high-speed runway exit manoeuvre. In the case where the aircraft follows the runway centerline, the cost function is comprised of the track cost and final attitude cost. The maximum tire/ground force is achieved on the inner gear. When considering the minimum time solution for the fast runway exit manoeuvre, an additional cost component in terms of the final distance to a target point was introduced. This causes the aircraft to exit the runway by cutting the corner at high speed. The tire/ground forces on the main gears are both close to their maximum level. The final optimal control sequence was for the case of minimum tire wear, where an additional cost associated with tire wear was added to the cost function. The result shows that the tire/ground forces for all the gears are kept at a lower level by using less steering and braking.

GOC provides a framework to optimize control strategies for aircraft ground manoeuvres. Future work could consider different runway geometries like 90-degree and 135-degree runway exits. The runway exit manoeuvres could also be extended to cover the taxiing phase to and from gates. In order to take advantage of commercial aircraft simulation software, GOC could be further developed to cope with black-box systems where no explicit 
system equations are available. Since GOC is an iterative algorithm and requires calculations in reverse time, it is suitable for off-line investigation rather than real-time implementation. However, the optimal solution given by GOC can also be used as a benchmark for real-time controller design.

\section{References}

[1] "Statistical Summary of Commercial Jet Airplane Accidents", Aviation Safety Boeing Commercial Airplanes P.O. Box 3707 M/C 0A-19 Seattle, Washington 98124-2207 USA.

[2] IATA, “Safety Report 2014”, 51st ed, April 2015, Montreal, ISBN 978-92-9252-582-8.

[3] J. Rankin, E. Coetzee, B. Krauskopf, M. Lowenberg, "Bifurcation and Stability Analysis of Aircraft Turning on the Ground", Journal of Guidance, Control, and Dynamics, Vol. 32, No. 2, March-April 2009

[4] J. Rankin, E. Coetzee, B. Krauskopf, M. Lowenberg, "Operational Parameter Study of Aircraft Dynamics on the Ground", Journal of Computational and Nonlinear Dynamics, April 2010, Vol. 5/021007-1.

[5] J. Duprez, F. Mora-Camino, F. Villaume, “Aircraft-on-ground lateral control for low speed manoeuvers”, in: Proceedings of the 16th IFAC Symposium on Automatic Control in Aerospace, St. Petersburg, Russia, 2004.

[6] C. Roos, J. Biannic, S. Tarbouriech, C. Prieur, M. Jeanneau, "On-ground aircraft control design using a parameter-varying anti-windup approach", Aerospace Science and Technology 14 (2010) 459-471

[7] C. Roos, J. Biannic, S. Tarbouriech, C. Prieur, "On-ground aircraft control design using an LPV anti-windup approach". In: Bates D, Hagstro“m M, editors. Nonlinear analysis and synthesis techniques for aircraft control, Vol. 365. Berlin Heidelberg: Springer Berlin Heidelberg; 2007. p. 117-45.

[8] Gordon, T.J. and Best, M.C. (2002) “A Sequential Dual Model Approach to Lap Optimisation”, Proceedings of the 6th International Symposium on Advanced Vehicle Control (AVEC), Hiroshima, Japan, pp.99-104.

[9] Gordon, T.J. and Best, M.C. (2007) "On the synthesis of driver inputs for the simulation of closed-loop handling manoeuvres", International Journal of Vehicle Design, Vol. 40, Nos. 1-3, pp.52-76.

[10] Best, M.C. (2012) "Optimisation of high-speed crash avoidance in autonomous vehicles", Int. J. Vehicle Autonomous Systems, Vol. 10, No. 4, pp.337-354.

[11] MathWorks, 2004, "Model and Simulate Mechanical Systems With SIMMECHANICS," http://www.mathworks.com/products/simmechanics/

[12] B. Etkin, "Dynamics of atmospheric flight”, Wiley, 1972.

[13] W. F. Phillips, “Mechanics of flight”, Wiley, January 2004.

[14] W. F. Milliken, D. L. Milliken, "Race Car Vehicle Dynamics", 1995, ISBN 1-56091-526-9.

[15] M. Blundell and D. Harty, “The multibody systems approach to vehicle dynamics”, SAE International, September 2004. 
[16] Bakker, E., L. Nyborg, and H. B. Pacejka, “Tire Modelling for Use in Vehicles Studies”, SAE Paper No. 870421, Society for Automotive Engineers, Warrendale, PA, 1987.

[17] Bakker, E., H. B. Pacejka, and L.Lidner, “A New Tire Model with an Application in Vehicle Dynamics Studies”, SAE Paper No. 890087, Society of Automotive Engineers, Warrendale, PA, 1989.

[18] Raivio T., Ehtamo H., Hämäläinen R.P. (1996) Aircraft trajectory optimization using nonlinear programming. In: Doležal J.,

Fidler J. (eds) System Modelling and Optimization. IFIP — The International Federation for Information Processing. Springer, Boston, MA

[19] Bryson, A.E. and Ho, Y.C. (1975) “Applied Optimal Control: Optimisation, Estimation and Control”, Hemisphere, New York.

[20] Marsh, C. (1992) “A Nonlinear Control Design Methodology for Computer-controlled Vehicle Suspension Systems”, PhD Thesis, Loughborough University.

[21] Jeanneau, M., 2004, “Description of Aircraft Ground Dynamics,” GARTEUR FM AG17, Paper No. RP0412731.

[22] Knisley, S. (2002). “A correlation between rolling tire contact friction energy and indoor tread wear". Tire Sci. and Technol. $30,2,83-99$.

[23] Veith, A. G. (1986). “The most complex tire-pavement interaction: Tire wear”. ASTM Special Technical Publication, 929, $125-158$.

[24] Fariba Fahroo and I. Michael Ross. "Direct Trajectory Optimization by a Chebyshev Pseudospectral Method", Journal of Guidance, Control, and Dynamics, Vol. 25, No. 1 (2002), pp. 160-166.

[25] Christopher L. Darby, William W. Hager, and Anil V. Rao. "Direct Trajectory Optimization Using a Variable Low-Order Adaptive Pseudospectral Method", Journal of Spacecraft and Rockets, Vol. 48, No. 3 (2011), pp. 433-445. 